\title{
Plasma Irradiation Effects to Intra- Abdominal Organs Compared with Adhesion Mouse Model
}

\author{
Keita Soda, ${ }^{\mathrm{a}, *}$ Hiroharu Yamashita, ${ }^{\mathrm{b}}$ Kyungho Chang, ${ }^{\mathrm{c}}$ Takeshi Kawamura, ${ }^{\mathrm{d}}$ \\ Takao Hamakubo ${ }^{a}$ \& Nobuyuki Shimizu ${ }^{e}$

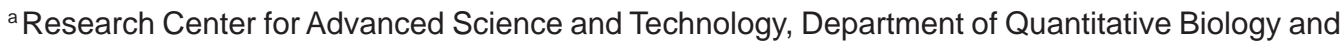 \\ Medicine, The University of Tokyo, Japan; 'bepartment of Gastrointestinal Surgery, Graduate \\ School of Medicine, University of Tokyo, Japan; 'The Anesthesiology and Pain Relief Center, \\ University of Tokyo Hospital, Japan; Isotope Science Center, The University of Tokyo, Japan; \\ eSanno Hospital, International University of Health and Welfare, Tokyo, Japan \\ *Address all correspondence to: Keita Soda, Department of Quantitative Biology and Medicine, Research Center for \\ Advanced Science and Technology, The University of Tokyo, 4-6-1 Komaba, Building 4, Room 317 Meguro-ku, \\ Meguro-city, Tokyo, Japan, 153-8904, E-mail: soda@qbm.rcast.u-tokyo.ac.jp
}

\begin{abstract}
In this study, we evaluated the effects of plasma irradiation on intra-abdominal organs using low-energy atmospheric-pressure plasma (LEAPP) compared to cauterization in the mouse adhesion model. Plasma irradiation of the cecum caused an increase of inflammatory proteins, such as serum amyloid proteins, inter- $\alpha$-trypsin inhibitor, and lactoferrin, in ascites. Plasma irradiation of the cecum and cauterization of the cecum induced mRNA expression of the pro-inflammatory cytokine IL-6. On the other hand, induction of lactoferrin in the cecum was lower with plasma irradiation than with cauterization. In addition, few inflammatory cells infiltrated the cecum after plasma irradiation compared with cauterization. We also demonstrated that very few adhesions formed after plasma irradiation compared to the mouse adhesion model. Furthermore, plasma irradiation is involved in IL-10 induction in blood plasma. Taken together, these results indicate that the reduced inflammatory effects of LEAPP should result in decreased formation of adhesions in tissues in the abdominal cavity.
\end{abstract}

KEY WORDS: plasma medicine, inflammation, peritoneal cavity, adhesion, ascites, proteomics

\section{INTRODUCTION}

Abdominal adhesion formation is an almost inevitable consequence of surgery (Fig. 1). ${ }^{1,2}$ Although intraperitoneal adhesion is not clearly identified without additional surgery, ${ }^{3}$ it is considered a very common outcome after surgery. ${ }^{4-7}$ Complications including small bowel obstruction, ${ }^{5}$ abdominal pain, ${ }^{6}$ and infertility in women ${ }^{7}$ are prevalent issues due to adhesion formation, and they sometimes requires inpatient hospital care, surgical intervention, or both, ${ }^{4,8}$ Therefore, this surgery-related event decreases the quality of life (QOL) of some patients.

Management and prevention of abdominal adhesion have attracted considerable concern by surgeons to reduce the need for later surgery due to their formation. ${ }^{3}$ When using a high frequency electric knife (HFEK) in surgery, cut mode or coagulation mode 

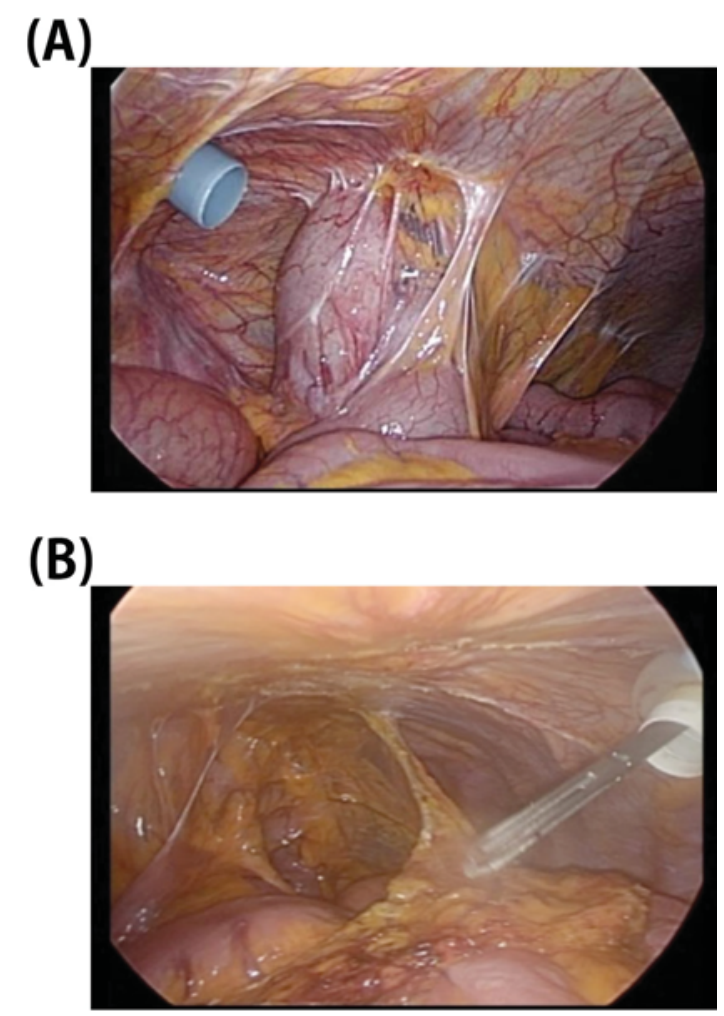

FIG. 1: Adhesion formation is secondary event after surgery. (a) Postoperative adhesions occur after almost every abdominal surgery and are the leading cause of intestinal obstruction. Adhesion formations between small intestine and abdominal wall were confirmed after surgery of gastric cancer. (b) Laparoscopic surgery for adhesiolysis is basically performed to cut the adhesion and separate the tissues, imposing a burden on patients.

generates the heat, which denatures tissue proteins, causes hemostasis, and thermally damages tissues. ${ }^{5,9}$ It might lead to leakage of plasma proteins, including fibrinogen and soluble fibrin at the site, which causes adhesion formation. ${ }^{10}$ Many investigators have taken on the challenges of preventing postoperative adhesion formation, ${ }^{3,11-13}$ and various types of polymer sheets have been developed in an aim to prevent adhesion formation. ${ }^{14-19}$ Separating the peritoneal surface around the wound from the organs by sodium hyaluronate/carboxymethylcellulose has been subjected to clinical trials and provided good results. ${ }^{20}$ However, location and pattern of adhesion formation are not limited to the tissue around the wound, and their development is basically unpredictable in the peritoneal cavity. ${ }^{3}$ The exact mechanism of how intraperitoneal adhesion formation is regulated is still unknown. ${ }^{3,11-13}$ Adhesion formation is regarded as a positive process in the wound healing to prevent invasion of infectious bacteria. In addition, adhesions may form regardless of the surgical devices used. ${ }^{21-23}$

Surgical devices that do not generate heat seem to be optimal to reduce the adhesion 
formation; however, such devices have not yet been developed. Low-energy atmospheric-pressure plasma (LEAPP) is a promising new tool in the medical field because of their physicochemical property, ${ }^{24-26}$ suggesting that it has greater potential than HFEKs. LEAPP does not generate the heat load, ${ }^{24-26}$ which could theoretically provide marginal thermal tissue injury. However, little is known about the effects of plasma irradiation on tissues in abdominal cavity. Moreover, the methods to identify and measure these effects have not been established. Therefore, finding the biological marker to validate the plasma irradiation effects on tissues quantitatively is the important initial step to employ LEAPP in surgical procedures. In this study, we evaluated these effects biologically using the mouse adhesion model.

\section{MATERIALS AND METHODS}

\section{A. Mice}

BALB/c, C57BL/6J, male or female mice aged 8 to 10 weeks (CLEA Japan, Inc.) were used. All animal experiments were performed in accordance with the guidelines of the Institutional Animal Care Committee of the University of Tokyo.

\section{B. Mouse Model of Surgic al Adhesion Formation}

We anesthetized mice with $3.6 \%$ isoflurane $(300 \mathrm{~mL} / \mathrm{min})$ using an aspirator (The Univentor 400 Anaesthesia Unit, Bioresearch Center Corporation, Japan) during the operation. We removed hair from the abdominal region of the mice by shaving to prevent contamination of the wound through contact with fur. Then the skin was disinfected with $70 \%$ ethanol. Then we made a small incision to open the abdomen using a small straight operating scissor. To optimize the adhesion formation model, we cauterized the abdominal wall, cecum, small intestine, or uterus, or multiple tissues using bipolar forceps (AARON 940 High Frequency Desiccator, Bovie Medical Corporation, USA). Alternatively, after conditions were optimized so that effects of cauterizing the cecum yielded reproducible results for the adhesion model, we located the cecum using blunt anatomical forceps inserted through a small incision and carefully moved it out of the peritoneal cavity so that mesenteric blood vessels would not be damaged. The cecum was cauterized using bipolar forceps and moved into the peritoneal cavity; then we closed the abdominal cavity using a surgical stapler. To confirm adhesion formation, we reopened the abdominal cavity at the indicated time points. We also performed sham surgeries and evaluated adhesion formation by adhesion score. ${ }^{27,28}$ Each mouse was evaluated according to the following standard scoring system: Score 0: no adhesion; score 1: filmy adhesion; score 2: more than one thin adhesion; score 3: thick adhesion with focal point; score 4: thick adhesion with plantar attachment or more than one thick adhesion with focal point; and score 5: very thick vascularized adhesion or more than one plantar adhesion.

Volume 7, Issue 4, 2017 


\section{Plasma Equipment and Plasma Inadiation}

We performed surgery on mice as well as cauterizing tissues. We carefully moved the cecum out of the peritoneal cavity, then irradiated the cecum with LEAPP (Dr. Sakakita kindly installed, The National Institute of Advanced Industrial Science and Technology [AIST], Japan), ${ }^{24,29}$ at one second per irradiation for a total of three times at the indicated discharge voltages. The flow rate of helium gas was set at two standard liters per minute, and the distance between the tip of the plasma jet and cecum was fixed at $10 \mathrm{~mm}$. We moved the cecum into the peritoneal cavity, then we closed the abdominal cavity using a surgical stapler. We reopened the abdominal cavity at the indicated time points to confirm whether adhesions had formed.

\section{Histological Analysis}

We reopened the postoperative peritoneal cavity and isolated the cecum and epididymal adipose tissue (EAT) by scissors, then put them into 10\% formalin for at least 24 hours. Subsequently, those tissues were placed in $70 \%$ ethanol solution until processing. After fixation, tissues were embedded in paraffin and prepared for paraffin block by the manufacturer (GenoStaff, Japan). The tissue was cut into $5 \mu \mathrm{m}$ sections for histological analysis. Samples were stained with hematoxylin and eosin (H\&E). The pathological sections were observed by optical microscope at x1, x50, and x400 magnifications.

\section{E. Proteomics Analysis of Asc ites}

We performed surgery on mice as described previously under continuous anesthesia, and we injected $1 \mathrm{~mL}$ phosphate buffered saline (PBS) into the closed abdominal cavity of the mice using a disposable 27-gauge needle to dilute the ascites with PBS. We massaged the abdominal region by pushing to mix well. Then we extracted $200 \mu \mathrm{L}$ peritoneal fluid from the abdomen using a 27 -gauge needle and dispensed $50 \mu \mathrm{L}$ for analysis following the procedure. Serum albumin and immunoglobulin in dispensed fluid were removed with the Albumin \& IgG Depletion Spin Trap (GE Healthcare Life Science, USA), and we prepared serum- and IgG-free samples. Furthermore, the sample was precipitated by trichloroacetic acid (TCA) for proteomics analysis. Finally, samples were trypsinized and analyzed by liquid chromatography-tandem mass spectrometry (LCMS/MS) system (LTQ Orbitrap Elite, Thermo Fisher Scientific K.K., USA). All MS/ MS datasets were analyzed by using Mascot software (version 2.5.1; Matrix Science Ltd., London, UK).

\section{F. Cytokine Assay}

After we performed each surgery, we collected $15 \mu \mathrm{L}$ blood from the tail vein at the indicated time points. Blood samples were then mixed with $20 \mu \mathrm{L}$ of $10 \mathrm{mg} / \mathrm{mL}$ EDTA 
to prevent coagulation. The blood was centrifuged at $1,000 \mathrm{xg}$ for $15 \mathrm{~min}$ at $4{ }^{\circ} \mathrm{C}$, and we harvested $15 \mu \mathrm{L}$ supernatant as plasma samples, which were stored at $-80^{\circ} \mathrm{C}$ until they were used for cytokine assays. Cytokines in plasma were then analyzed using a BioPlex (Bio-Rad Laboratories, USA) bead-based system, which was designed to quantitate multiple cytokines in diverse matrices. We also assessed IL-6 and IL-10 cytokine levels in blood plasma individually with IL-6 and IL-10 ELISA kits (Thermo Fisher Scientific K.K., USA).

\section{G. Quantitative PCR of Local Tissue}

We sectioned the cecum at one-third of the distance from the tip where the cauterized or irradiated region was located; then samples were placed in $500 \mu \mathrm{L}$ RNAlater (Thermo Fisher Scientific K.K., USA) to stabilize RNA in the tissue. To isolate total RNA from cecum samples, we first removed all feces from the cecum in a dish and washed the cecum by dipping repeatedly in in 6-well plates filled with PBS filled. Cecum samples were then homogenized on ice in Buffer RLT (Qiagen, Germany) using a Dounce homogenizer (AS ONE, Japan). The homogenate was then centrifuged at 15,000 $\mathrm{x} g$ for 3 min, and the supernatant was harvested as lysate including RNA. The lysate was mixed with an equal amount of $70 \%$ ethanol, and then the mixture was applied to an RNeasy spin column (Qiagen, Germany). We finally extracted total RNA according to the manufacturer's protocol (RNeasy Mini Kit, Qiagen, Germany). mRNA was reverse transcribed to cDNA with the iScript cDNA Synthesis Kit (Bio-Rad Laboratories, USA). Target genes were quantitatively amplified by CFX96 Real-Time System (Bio-Rad Laboratories, USA). The following primers were used.

IL-6 (Forward 5'-AGTTGCCTTCTTGGGACTGA-3', Reverse 5'-CAGAATTGCCATTGCACAAC-3')

Lactoferrin (Forward 5'-GGAGCCTTGAGGTGTCTGAG-3', Reverse 5'-CCAGGTGGCACTCCTTGTAT-3')

Cyclophilin(Forward 5'-TTCGTGCTCTGAGCACTGGAGA-3', Reverse 5'-GGACCCGTATGCTTTAGGATGAAG-3')

\section{H. Statistic al Analysis}

Data are expressed as mean \pm SEM and were analyzed using GraphPad Prism 7 statistical software (GraphPad Software, California, USA). Significant difference was determined by multiple $t$-tests.

Volume 7, Issue 4, 2017 


\section{RESULTS}

\section{A. Stably Occ urred Abdominal Adhesion Mouse Model Was Generated}

Some mouse adhesion models have been reported, such as ischemic buttons to peritoneal sidewall, ${ }^{30}$ the multiple-ablation model using a toothbrush, ${ }^{31}$ and cauterization of the cecum using the coagulation mode of bipolar forceps.$^{28}$ Since bipolar forceps are a generally and widely used surgical device, we chose to generate a mouse abdominal adhesion model using bipolar forceps. First, we assessed the severity of adhesion formation by adhesion score and reproducibility of adhesion formation in case of cauterizing various tissues, changing the power of the bipolar forceps, and using mice of different sexes and ages. Thus, cauterizing the cecum of two-month-old male mice yielded formation of reproducible adhesions. We set the output power of the bipolar forceps to 3 $\mathrm{W}$ and cauterized the cecum three times for $1 \mathrm{sec}$ each time at different areas(Fig. 2[a]), then put the cecum back into the peritoneal cavity. We confirmed that adhesions formed as early as one day after surgery. Adhesion formation occurred mainly between the cecum and epididymal adipose tissue (EAT), and they remained for 5 months after cauterization (Fig. 2[b]). Body weight change was not significantly different between sham and cauterized mice for seven days after surgery (Fig. 2[c]). Adhesions that formed in our model were flaccid or intermediate adhesions and not fibrotic (Fig. 2[d]).

\section{B. Plasma Inadiation Showed Different Effects Compared to Cauterization}

Next, we applied LEAPP irradiation (Fig. 3[a]) to the cecum three times for $1 \mathrm{sec}$ each at the indicated discharge voltages (Fig. $3[\mathrm{~b}, \mathrm{c}]$ ). These conditions yielded almost the same effects as well as cauterizing the cecum, and they were sufficient to coagulate blood. Plasma irradiation to the cecum at $14 \mathrm{~V}$ or $16 \mathrm{~V}$ discharge voltage resulted in adhesion formation with great omentum, not EAT, which was a very weak adhesion compared to those induced by cauterizing the cecum (Fig. 3[d,e]). Adhesion score was almost 0 or 1 . On the other hand, there was no adhesion formation in the abdominal cavity when the voltage was set at $18 \mathrm{~V}$.

\section{Infiltration of Inflammatory Cells Was Observed in Plasma-Irradiated Area}

To assess tissue inflammation caused by plasma irradiation, we performed histopathological examination by H\&E staining of sham, cauterized cecum, and plasma-irradiated cecum. Inflammatory cells including neutrophils and macrophages strongly infiltrated the submucosal layer beneath the cauterized area (Fig. 4[b]). On the other hand, there was infiltration of a few inflammatory cells in the submucosal layer beneath the plasmairradiated area (Fig. 4[c]). In addition, we verified inflammatory cytokine levels in blood. Among tested cytokines, pro-inflammatory interleukin-6 (IL-6) and anti-inflammatory 
(A)

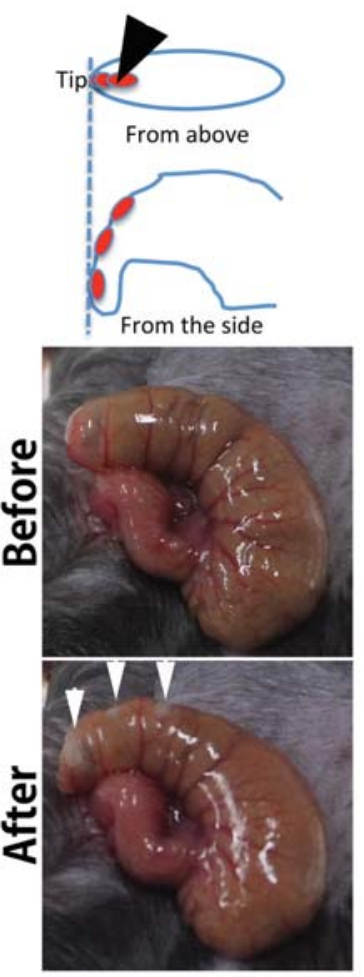

(C)

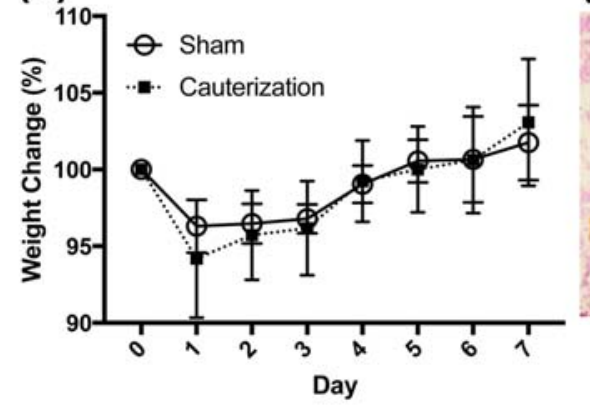

(B) Sham Cauterization

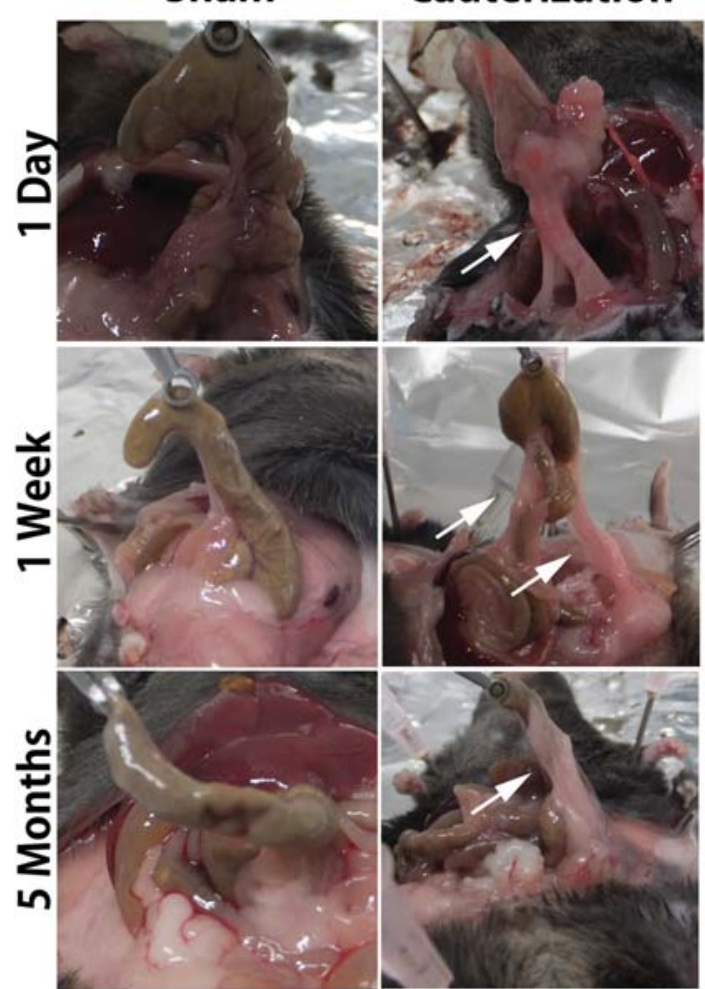

(D)

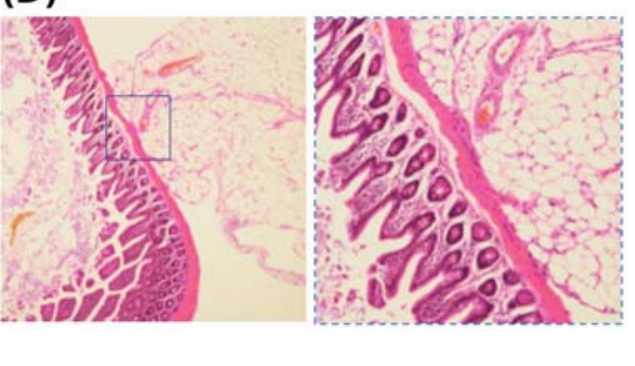

FIG. 2: Cauterizing cecum results in adhesion formation. (a) We optimized conditions to generate adhesion formation in mouse peritoneal cavity. The cecum was cauterized at three points by using bipolar forceps (set $3 \mathrm{~W}$ output power, indicated by black arrow) were tanned on the surface of cecum (white arrow) and were put back into the peritoneal cavity. (b) White arrow indicates adhesion formation between cecum and epididymal adipose tissue. Presence of adhesions was confirmed as early as one day and still confirmed at five months after operation. (c) No significant weight changes were confirmed between sham and cauterized mouse $(N=6)$. $(\mathrm{d})$ Adipose tissue formed adhesions with the cecum that were firm, not fibrotic, and steady. Inflammatory cells were also confirmed in adipose tissue, especially on the adhesion formation side. 
(A)

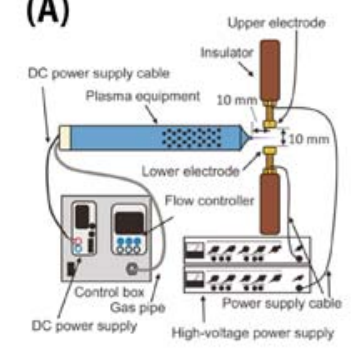

(B)

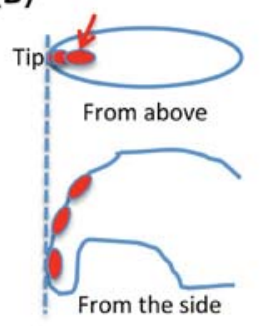

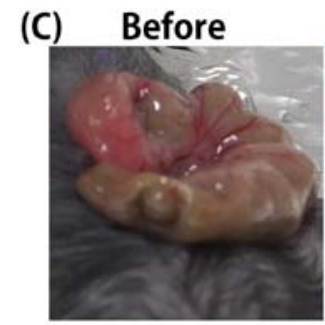

(D)
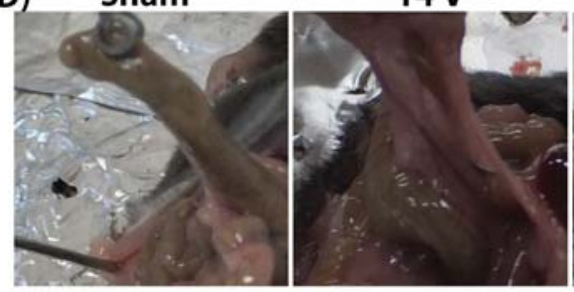

(E)

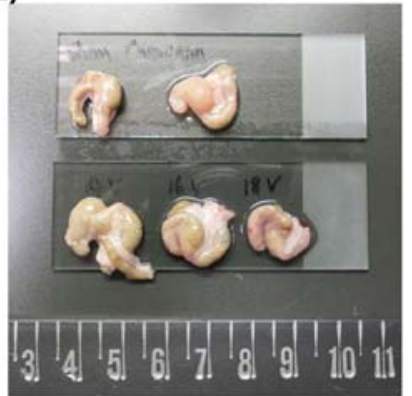

FIG. 3: Plasma irradiation on cecum demonstrates no adhesion or adhesion with omentum. (a) Low-energy atmospheric-pressure plasma (LEAPP) equipment we used was installed in our laboratory by Dr. Sakakita (AIST, Japan). Helium was used as the carrier gas. (b), (c) Plasma produced in the quartz tube and ejected from the nozzle exit into the surrounding gas was irradiated on cecum in the same manner as cauterizing. (d) Plasma irradiation set by DC voltage of $14 \mathrm{~V}$, $16 \mathrm{~V}$, or $18 \mathrm{~V}$ resulted in adhesion formation with omentum or no adhesion formation, indicating different effects of cauterizing the cecum. (e) When DC voltage was set at $18 \mathrm{~V}$, no adhesion formation was confirmed. Following the experiment, we set the DC voltage at $18 \mathrm{~V}$. 
(A)
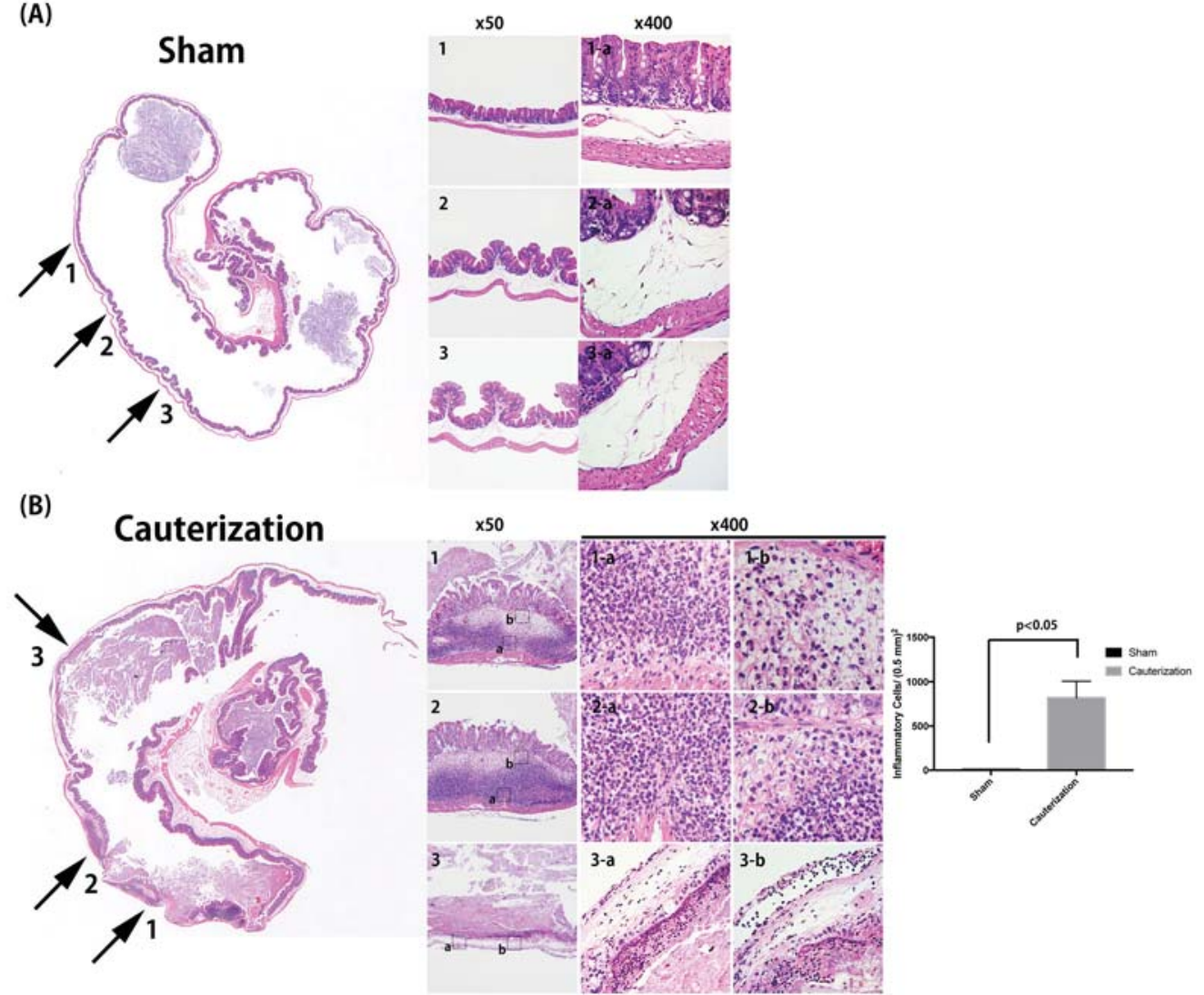

(C)
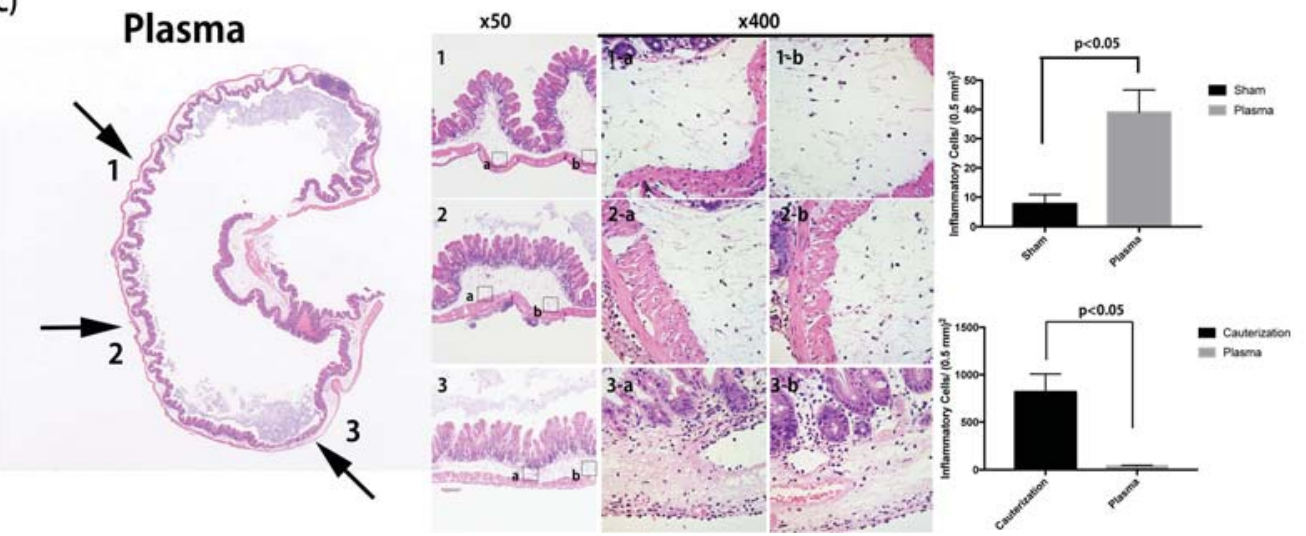

FIG. 4: H\&E staining demonstrates the inflammatory infiltrate and plasma irradiation on tissue induced inflammatory cells not so much as cauterizing tissue. (a) No inflammatory cells were confirmed in the sham controls. (b) The inflammatory cells infiltrate in submucosa extending up to the mucosa in cauterized cecum. (c) Submucosal inflammation was present in plasma-irradiated cecum, but there were a few inflammatory cells compared to (b). Statistical data shown on the right was representative of $\mathrm{x} 400$ images $(N=4)$. 
interleukin-10 (IL-10) showed significant changes after cauterization of cecum, as shown in Fig. 5 ( $p<0.05)$. Plasma irradiation induced IL-6 at 9 hours, whereas the IL-6 concentration after plasma irradiation was significantly lower than the IL-6 level associated with cauterization at that time. IL-10 induced by plasma irradiation showed a similar trend, with IL-10 induction by cauterization at 3, 9, and 24 hours. IL-10 concentration induced by plasma irradiation was slightly higher than IL-10 concentration induced by cauterization ( $p<0.5$ at 3 hours; $p<0.5$ at 9 hours; $p<0.5$ at 24 hours) (Fig. 6).

\section{Proteomics Analysis Identified Inflammatory Proteins in Ascites}

Furthermore, we performed comprehensive proteomics analysis of ascites for identifying specific inflammatory proteins after surgery in time-dependent manner to determine whether any inflammatory mediators are released from damaged cecum. As shown in Table 1, some inflammatory proteins were identified, such as lactoferrin, serum amyloid protein, inter- $\alpha$-trypsin inhibitor, serine protease inhibitor, and apolipoprotein B, compared with sham. As shown in Tables 2 and 3, there were almost the same inflammatory proteins in ascites after both plasma irradiation and cauterization.

\section{E. Quantitative Analysis Revealed that Plasma Imadiation on Local Tissue Exerts Lower Inflammatory Effects}

Lactoferrin was identified in both cauterized and plasma irradiated cecum. Lactoferrin is known to be released from neutrophils. ${ }^{32}$ As Fig. 4 shows, a number of neutrophils infiltrating the submucosa was significantly different after cauterizing the cecum compared to plasma irradiation. Therefore, we assessed mRNA expression level using cauterized cecum or plasma-irradiated cecum in time-dependent manner. We confirmed the presence of lactoferrin mRNA using specific primers as well as the inflammatory marker IL-6, as shown in blood (Fig. 6). We demonstrated that plasma irradiation to cecum induces production of IL-6 as well as cauterizing cecum, whereas plasma irradiation did not induce lactoferrin significantly, compared to cauterization to cecum (Fig. 7).

\section{DISCUSSION}

Low-energy atmospheric-pressure plasma (LEAPP) has been suggested to be beneficial in wound healing ${ }^{24,33-36}$ and is expected to be useful as a surgical device. ${ }^{34,35,37}$ The latter may reduce postoperative problems, such as adhesion formation. However, the effect of LEAPP on abdominal organs is not well understood. Therefore, we evaluated the effects of plasma irradiation and compared them to the mouse adhesion model, induced by cauterization in this study. Proteomics of ascites demonstrated the elevation of inflammatory proteins in ascites after plasma irradiation of the cecum as well as cauterization of the cecum (Tables 1, 2, and 3). These results did not show obvious differences between plasma irradiation and cauterization of the cecum. On the other hand, histological examinations revealed obvious differences, including fewer infiltrated inflammatory cells 

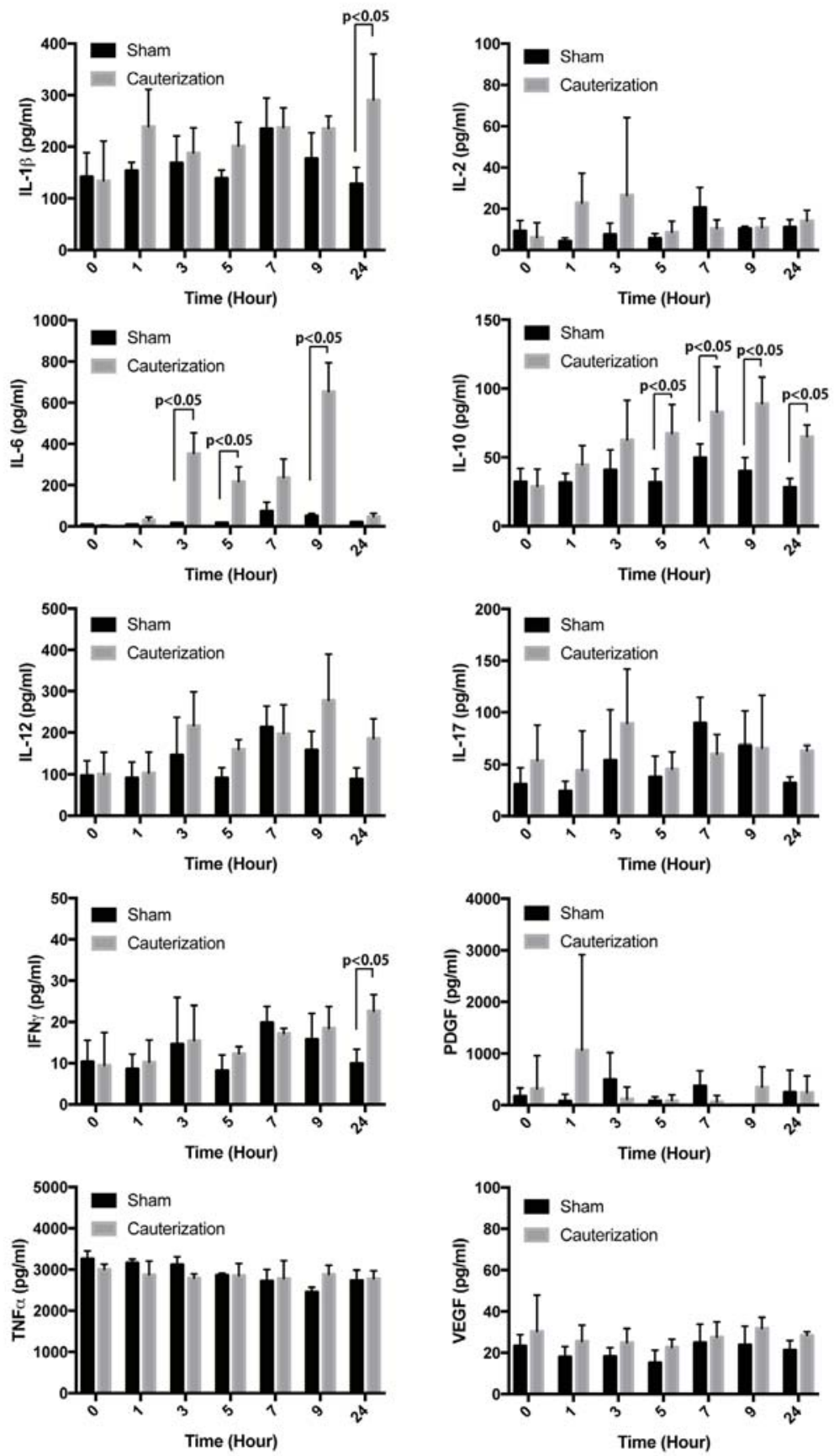

FIG. 5: IL-6 and IL-10 were induced after cauterization in plasma. Cytokine levels in plasma were assessed using Bio-Plex. Inflammatory cytokine IL-6 were induced significantly, and proinflammatory cytokine IL-10 was also induced after cauterization. ( $N=3$ in each experiment). 

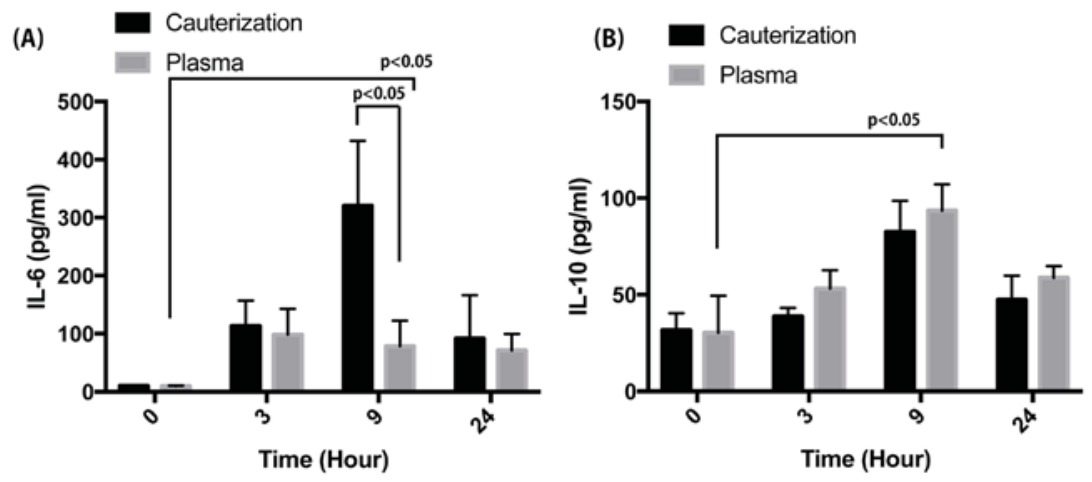

FIG. 6: IL-6 in plasma was not induced after plasma irradiation as much as in cauterization. (a) IL-6 was induced by plasma irradiation of the cecum, but the level was much lower than that after cauterization. (b) The level of IL-10 was induced by plasma irradiation as much as cauterizing the cecum. ( $N=4$ in each experiment).

in the submucosal layer of plasma-irradiated cecum. However, massive infiltration of the inflammatory cells was observed in the submucosal layer of cauterized cecum (Fig. 4). To assess the inflammation of the local tissue by plasma irradiation and cauterization, we quantitatively assessed the mRNA expression levels related to inflammation in local areas of the cecum (Fig. 7). Hence, increased mRNA level of pro-inflammatory cytokine IL-6 was observed in plasma-irradiated cecum as well as cauterized cecum (Fig. 7[a]), indicating that plasma irradiation and cauterization of the cecum induce comparable inflammatory responses. Induction of IL-6 reveals that plasma irradiation should be a causative stimulus to non-inflammatory cells, such as endothelial cells ${ }^{38,39}$ and fibroblasts ${ }^{40,41}$ which are known to produce IL-6 in response to inflammatory stimulation. On the other hand, lactoferrin mRNA expression, known to be produced by neutrophils, ${ }^{42}$ was not increased by plasma irradiation compared with cauterization, which was in response to infiltrated inflammatory cells observed in submucosal layer. In contrast, the increase of IL- 6 concentration in blood plasma at 9 hours after cauterization is presumed by systemic inflammatory response to cauterizing cecum and this plasma irradiation effect was weak. We also demonstrated that plasma irradiation as well as cauterization induced anti-inflammatory cytokine IL-10 in blood plasma. Of note, IL-10 induced by plasma irradiation to cecum was slightly higher than IL-10 induced by cauterization to cecum at 3,9 , and 24 hours $(P<0.5)$. These data indicated a reduced inflammatory effect of plasma irradiation compared with cauterization and suggest that cytokine balance due to plasma irradiation is involved in wound healing, ${ }^{43,44}$ leading to a decrease in adhesion formation. ${ }^{21,23}$ 
TABLE 1: Peritoneal ascites were analyzed by proteomics. Proteomics of ascites from the peritoneal cavity of sham controls, treated with cauterization or plasma irradiation to the cecum, identified some inflammatory proteins. In Tables $1-3$, gray protein names indicate proteins identified in both cauterization and plasma irradiation. The number indicates identified peptides.

\section{Sham}

\section{Cauterization}

\begin{tabular}{lcccccccc}
\multicolumn{1}{c}{ Accession Number } & $\mathbf{0} \mathbf{h r}$ & $\mathbf{0 . 5}$ & $\mathbf{4}$ & $\mathbf{2 4}$ & $\mathbf{0} \mathbf{h r}$ & $\mathbf{0 . 5} \mathbf{h r}$ & $\mathbf{4} \mathbf{h r}$ & $\mathbf{2 4}$ \\
A1AG2 & 1 & 1 & 1 & 1 & 0 & 1 & 1 & 4 \\
\hline ENOB & 0 & 5 & 2 & 2 & 0 & 7 & 6 & 0 \\
CHIL3 & 0 & 0 & 1 & 1 & 0 & 1 & 6 & 2 \\
FRIH & 1 & 5 & 4 & 1 & 0 & 0 & 1 & 2 \\
\hline FRIL1 & 0 & 8 & 1 & 0 & 0 & 2 & 0 & 1 \\
\hline G6PI & 1 & 5 & 4 & 1 & 0 & 4 & 2 & 0 \\
G3P & 2 & 0 & 0 & 4 & 0 & 2 & 0 & 0 \\
LAC2 (+1) & 4 & 2 & 2 & 0 & 0 & 0 & 0 & 0 \\
K1C16 & 0 & 0 & 0 & 3 & 0 & 0 & 0 & 0 \\
K1C17 & 0 & 1 & 8 & 14 & 0 & 0 & 0 & 0 \\
K1C42 & 2 & 1 & 2 & 5 & 0 & 4 & 0 & 0 \\
K2C73 & 3 & 2 & 2 & 3 & 0 & 0 & 0 & 0 \\
TRFL & 0 & 0 & 4 & 6 & 0 & 7 & 33 & 17 \\
\hline MYH1 (+1) & 0 & 3 & 0 & 0 & 0 & 0 & 1 & 0 \\
MYH4 & 0 & 0 & 27 & 9 & 0 & 0 & 15 & 10 \\
\hline PGM1 & 1 & 2 & 1 & 0 & 1 & 0 & 2 & 3 \\
S10A9 & 0 & 5 & 1 & 0 & 0 & 1 & 0 & 0 \\
SAA1 & 0 & 0 & 5 & 0 & 0 & 0 & 0 & 0 \\
SAA2 & 2 & 0 & 6 & 1 & 1 & 0 & 0 \\
\hline SAA3 & 0 & 1 & 2 & 0 & 1 & 0 & 0 & 0 \\
\hline TPM2 & 0 & 2 & 4 & 0 & 4 & 7 & 6 \\
ENOA & 0 & 0 & 0 & 0 & 0 & 9 & 11 & 9 \\
ACE & 0 & 0 & 0 & 0 & 0 & 1 & 5 & 1 \\
CBPQ & 0 & 0 & 0 & 0 & 0 & 0 & 0 & 3 \\
CO1A1 & 0 & & & & & & \\
\hline & 0 & 3 & 2 & 1 & 0 & 1 & 0 \\
\hline
\end{tabular}


TABLE 1: (continued)

\begin{tabular}{|c|c|c|c|c|c|c|c|c|}
\hline KCRM & 0 & 28 & 2 & 9 & 1 & 25 & 8 & 0 \\
\hline FBLN3 & 0 & 0 & 0 & 0 & 1 & 0 & 0 & 3 \\
\hline SODE & 1 & 0 & 0 & 0 & 1 & 0 & 1 & 3 \\
\hline HA10 & 0 & 0 & 1 & 0 & 1 & 1 & 1 & 4 \\
\hline K1C14 & 0 & 0 & 0 & 3 & 1 & 0 & 1 & 0 \\
\hline K2C5 & 0 & 1 & 2 & 6 & 1 & 1 & 1 & 0 \\
\hline K2C6A & 1 & 4 & 7 & 14 & 1 & 2 & 5 & 0 \\
\hline LDHA & 2 & 4 & 4 & 1 & 1 & 4 & 4 & 1 \\
\hline MUP1 & 16 & 13 & 1 & 14 & 1 & 1 & 1 & 1 \\
\hline PI16 & 3 & 0 & 0 & 0 & 1 & 0 & 1 & 1 \\
\hline PGK1 & 0 & 8 & 1 & 1 & 1 & 6 & 1 & 0 \\
\hline KРYM & 3 & 12 & 13 & 3 & 1 & 9 & 12 & 4 \\
\hline SEPP1 & 1 & 1 & 1 & 0 & 1 & 3 & 4 & 5 \\
\hline SAMP & 1 & 1 & 0 & 1 & 1 & 6 & 6 & 8 \\
\hline QSOX1 & 0 & 0 & 0 & 0 & 1 & 3 & 5 & 4 \\
\hline AMY1 & 2 & 2 & 2 & 1 & 2 & 1 & 1 & 3 \\
\hline CRP & 2 & 0 & 2 & 2 & 2 & 2 & 3 & 3 \\
\hline CAH3 & 2 & 16 & 7 & 6 & 2 & 13 & 9 & 1 \\
\hline KV2A7 & 3 & 2 & 2 & 2 & 2 & 2 & 0 & 0 \\
\hline IGJ & 4 & 3 & 3 & 2 & 2 & 2 & 2 & 1 \\
\hline ITIH4 & 0 & 0 & 0 & 0 & 2 & 11 & 14 & 20 \\
\hline $\mathrm{K} 2 \mathrm{C} 1$ & 1 & 1 & 2 & 2 & 2 & 3 & 2 & 1 \\
\hline PPIA & 2 & 2 & 2 & 1 & 2 & 3 & 0 & 2 \\
\hline SAA4 & 4 & 2 & 2 & 1 & 2 & 2 & 4 & 6 \\
\hline TPIS & 2 & 8 & 5 & 2 & 2 & 6 & 4 & 0 \\
\hline $\mathrm{CAH} 2$ & 6 & 4 & 4 & 2 & 3 & 6 & 3 & 4 \\
\hline CATB & 3 & 1 & 4 & 2 & 3 & 1 & 2 & 3 \\
\hline CD5L & 4 & 3 & 5 & 2 & 3 & 1 & 3 & 3 \\
\hline F13B & 0 & 0 & 1 & 0 & 3 & 0 & 4 & 3 \\
\hline CS1A & 1 & 0 & 1 & 1 & 3 & 2 & 2 & 1 \\
\hline
\end{tabular}


TABLE 1: (continued)

\begin{tabular}{|c|c|c|c|c|c|c|c|}
\hline HEP2 & 0 & 0 & 0 & 0 & 3 & 1 & 2 \\
\hline IGKC & 6 & 5 & 4 & 3 & 3 & 3 & 2 \\
\hline MUP2 & 3 & 3 & 3 & 2 & 3 & 1 & 3 \\
\hline MUP3 & 3 & 2 & 4 & 2 & 3 & 1 & 1 \\
\hline RET4 & 0 & 0 & 1 & 0 & 3 & 0 & 3 \\
\hline SPA3M & 2 & 1 & 1 & 1 & 3 & 9 & 16 \\
\hline SPA3N & 2 & 0 & 2 & 1 & 3 & 18 & 27 \\
\hline ADIPO & 4 & 2 & 4 & 2 & 4 & 4 & 4 \\
\hline A1AT1 & 4 & 2 & 10 & 2 & 4 & 14 & 4 \\
\hline APOB & 0 & 0 & 8 & 0 & 4 & 14 & 32 \\
\hline CBPN & 4 & 2 & 5 & 3 & 4 & 4 & 6 \\
\hline LUM & 4 & 3 & 4 & 3 & 4 & 3 & 5 \\
\hline MUP20 & 5 & 5 & 5 & 3 & 4 & 1 & 2 \\
\hline PROP & 8 & 4 & 8 & 0 & 4 & 6 & 9 \\
\hline PON1 & 4 & 3 & 5 & 2 & 4 & 5 & 5 \\
\hline ANGT & 4 & 4 & 5 & 3 & 5 & 7 & 6 \\
\hline CBPB2 & 7 & 8 & 8 & 7 & 5 & 8 & 8 \\
\hline CO8G & 2 & 1 & 3 & 1 & 5 & 5 & 4 \\
\hline GPX3 & 3 & 1 & 4 & 2 & 5 & 3 & 3 \\
\hline HBA & 9 & 5 & 5 & 5 & 5 & 12 & 7 \\
\hline IGHA & 7 & 3 & 6 & 1 & 5 & 2 & 5 \\
\hline IL1AP & 2 & 0 & 3 & 0 & 5 & 3 & 4 \\
\hline AMBP & 4 & 2 & 2 & 2 & 5 & 5 & 6 \\
\hline ZPI & 1 & 2 & 3 & 1 & 5 & 7 & 3 \\
\hline APOA2 & 6 & 4 & 5 & 6 & 6 & 6 & 6 \\
\hline FA12 & 2 & 1 & 6 & 0 & 6 & 4 & 7 \\
\hline $\mathrm{CO} 8 \mathrm{~B}$ & 4 & 0 & 6 & 0 & 6 & 7 & 11 \\
\hline ALS & 5 & 6 & 5 & 3 & 6 & 4 & 7 \\
\hline ZA2G & 1 & 0 & 1 & 0 & 6 & 3 & 3 \\
\hline A2AP & 2 & 0 & 2 & 0 & 7 & 2 & 6 \\
\hline
\end{tabular}


TABLE 1: (continued)

\begin{tabular}{|c|c|c|c|c|c|c|c|c|}
\hline CLUS & 5 & 3 & 7 & 2 & 7 & 6 & 8 & 9 \\
\hline ALDOA & 5 & 17 & 20 & 7 & 7 & 20 & 19 & 6 \\
\hline HBB 1 & 10 & 6 & 4 & 4 & 7 & 10 & 8 & 9 \\
\hline ITIH3 & 0 & 0 & 2 & 0 & 7 & 6 & 13 & 21 \\
\hline PEDF & 8 & 6 & 7 & 3 & 7 & 5 & 5 & 7 \\
\hline IC 1 & 5 & 1 & 5 & 2 & 7 & 4 & 7 & 6 \\
\hline ACTB (+1) & 5 & 4 & 6 & 6 & 8 & 15 & 7 & 10 \\
\hline CPN2 & 2 & 3 & 6 & 3 & 8 & 7 & 6 & 8 \\
\hline CO9 & 2 & 0 & 1 & 0 & 8 & 3 & 5 & 6 \\
\hline CFAD & 8 & 7 & 7 & 7 & 8 & 7 & 6 & 6 \\
\hline $\mathrm{CBG}$ & 6 & 8 & 7 & 5 & 8 & 7 & 6 & 5 \\
\hline HPT & 11 & 8 & 11 & 9 & 8 & 21 & 20 & 19 \\
\hline PHLD & 3 & 1 & 4 & 2 & 8 & 8 & 7 & 10 \\
\hline VTNC & 6 & 1 & 8 & 1 & 8 & 10 & 9 & 10 \\
\hline A1AT3 & 7 & 6 & 0 & 6 & 9 & 0 & 8 & 10 \\
\hline APOH & 8 & 3 & 10 & 3 & 9 & 9 & 16 & 11 \\
\hline THRB & 4 & 0 & 3 & 1 & 10 & 7 & 13 & 12 \\
\hline FETUB & 6 & 3 & 4 & 4 & 11 & 8 & 11 & 10 \\
\hline LIFR & 11 & 3 & 10 & 2 & 11 & 3 & 6 & 4 \\
\hline AMYP & 6 & 9 & 7 & 2 & 11 & 1 & 5 & 0 \\
\hline APOE & 8 & 7 & 13 & 8 & 12 & 16 & 22 & 16 \\
\hline CO8A & 3 & 1 & 4 & 0 & 12 & 10 & 7 & 12 \\
\hline ITIH1 & 3 & 1 & 5 & 1 & 12 & 5 & 6 & 11 \\
\hline A1AG1 & 15 & 13 & 12 & 11 & 13 & 14 & 15 & 18 \\
\hline KLKB1 & 4 & 0 & 8 & 1 & 13 & 12 & 13 & 11 \\
\hline A1AT5 & 14 & 9 & 12 & 11 & 14 & 14 & 15 & 17 \\
\hline FETUA & 14 & 7 & 11 & 5 & 14 & 15 & 18 & 16 \\
\hline CFAI & 9 & 2 & 7 & 4 & 14 & 13 & 15 & 12 \\
\hline EGFR & 9 & 2 & 9 & 2 & 14 & 14 & 14 & 14 \\
\hline ITIH2 & 5 & 0 & 3 & 2 & 14 & 12 & 14 & 17 \\
\hline
\end{tabular}


TABLE 1: (continued)

\begin{tabular}{|c|c|c|c|c|c|c|c|c|}
\hline MUP6 & 2 & 2 & 16 & 2 & 14 & 8 & 11 & 6 \\
\hline TTHY & 14 & 15 & 12 & 13 & 14 & 15 & 14 & 15 \\
\hline A1AT2 & 45 & 17 & 14 & 15 & 16 & 18 & 47 & 46 \\
\hline $\mathrm{CO} 5$ & 7 & 1 & 17 & 3 & 16 & 17 & 16 & 20 \\
\hline IGHM & 29 & 27 & 25 & 18 & 16 & 16 & 18 & 11 \\
\hline HRG & 11 & 4 & 13 & 4 & 17 & 16 & 19 & 17 \\
\hline SPA3K & 15 & 16 & 14 & 16 & 18 & 17 & 8 & 17 \\
\hline APOA4 & 18 & 4 & 18 & 7 & 20 & 22 & 24 & 20 \\
\hline KNG1 & 13 & 7 & 14 & 8 & 20 & 19 & 19 & 24 \\
\hline FIBG & 16 & 11 & 16 & 11 & 21 & 17 & 29 & 0 \\
\hline ICA & 17 & 7 & 13 & 5 & 21 & 19 & 18 & 23 \\
\hline AFAM & 22 & 23 & 23 & 15 & 22 & 25 & 28 & 25 \\
\hline FIBA & 28 & 17 & 21 & 17 & 26 & 29 & 39 & 3 \\
\hline GELS & 13 & 6 & 12 & 7 & 26 & 23 & 16 & 21 \\
\hline FIBB & 25 & 13 & 20 & 9 & 28 & 28 & 35 & 0 \\
\hline ANT3 & 18 & 14 & 16 & 15 & 29 & 28 & 18 & 22 \\
\hline ALBU & 8 & 8 & 27 & 11 & 30 & 28 & 26 & 16 \\
\hline CFAB & 26 & 13 & 24 & 13 & 32 & 29 & 34 & 42 \\
\hline APOA1 & 37 & 26 & 30 & 27 & 34 & 36 & 36 & 40 \\
\hline EST1C & 32 & 29 & 31 & 27 & 35 & 33 & 31 & 29 \\
\hline PLMN & 15 & 6 & 16 & 4 & 35 & 32 & 38 & 42 \\
\hline $\mathrm{CO} 4 \mathrm{~B}$ & 3 & 1 & 8 & 2 & 36 & 30 & 27 & 36 \\
\hline HEMO & 40 & 33 & 38 & 27 & 37 & 39 & 38 & 44 \\
\hline CFAH & 36 & 19 & 37 & 17 & 40 & 40 & 60 & 57 \\
\hline A1AT4 & 17 & 39 & 37 & 38 & 42 & 44 & 17 & 17 \\
\hline VTDB & 38 & 25 & 30 & 26 & 44 & 47 & 44 & 37 \\
\hline FINC & 30 & 18 & 30 & 8 & 45 & 42 & 48 & 37 \\
\hline CERU & 62 & 32 & 53 & 45 & 59 & 63 & 62 & 63 \\
\hline MUG1 & 56 & 37 & 50 & 36 & 63 & 69 & 66 & 66 \\
\hline $\mathrm{A} 2 \mathrm{M}$ & 102 & 77 & 92 & 77 & 92 & 102 & 107 & 106 \\
\hline
\end{tabular}


TABLE 1: (continued)

\begin{tabular}{|c|c|c|c|c|c|c|c|c|}
\hline TRFE & 117 & 105 & 91 & 105 & 107 & 109 & 110 & 111 \\
\hline $\mathrm{CO} 3$ & 79 & 52 & 91 & 64 & 118 & 117 & 123 & 136 \\
\hline
\end{tabular}

TABLE 2: Peritoneal ascites were analyzed by proteomics

Sham

Plasma (1)

Accession Number

$$
1433 Z
$$

ACTS (+1)

ACTA (+1)

$\operatorname{ACTB}(+1)$

$\mathrm{A} 1 \mathrm{AG} 2$

A1AT3

A2M

AMY1

ANXA1

APOB

APOC3

APOD

APOM

C4BPA

\section{CBPN}

CPN2

CD5L

CHIL3

CLUS

FA10

F13A

CO1A1

CO3A1

\begin{tabular}{|c|c|c|c|c|c|c|c|}
\hline \multicolumn{4}{|c|}{ Sham } & \multicolumn{4}{|c|}{ Plasma (1) } \\
\hline $0 \mathrm{hr}$ & $0.5 \mathrm{hr}$ & $4 \mathrm{hr}$ & $24 \mathrm{hr}$ & $0 \mathrm{hr}$ & $0.5 \mathrm{hr}$ & $4 \mathrm{hr}$ & $24 \mathrm{hr}$ \\
\hline 0 & 0 & 0 & 0 & 0 & 3 & 0 & 2 \\
\hline 0 & 0 & 2 & 1 & 0 & 0 & 3 & 0 \\
\hline 0 & 0 & 0 & 0 & 0 & 2 & 0 & 4 \\
\hline 5 & 4 & 6 & 6 & 0 & 15 & 9 & 19 \\
\hline 1 & 1 & 1 & 1 & 0 & 0 & 0 & 3 \\
\hline 7 & 6 & 0 & 6 & 0 & 0 & 7 & 0 \\
\hline 102 & 77 & 92 & 77 & 0 & 0 & 0 & 0 \\
\hline 2 & 2 & 2 & 1 & 0 & 3 & 3 & 2 \\
\hline 0 & 0 & 0 & 0 & 0 & 0 & 5 & 0 \\
\hline 0 & 0 & 8 & 0 & 0 & 24 & 10 & 39 \\
\hline 2 & 2 & 2 & 2 & 0 & 3 & 2 & 3 \\
\hline 1 & 0 & 1 & 0 & 0 & 3 & 2 & 3 \\
\hline 0 & 0 & 0 & 0 & 0 & 3 & 2 & 3 \\
\hline 2 & 0 & 1 & 1 & 0 & 7 & 4 & 10 \\
\hline 4 & 2 & 5 & 3 & 0 & 10 & 8 & 10 \\
\hline 2 & 3 & 6 & 3 & 0 & 12 & 10 & 13 \\
\hline 4 & 3 & 5 & 2 & 0 & 7 & 4 & 8 \\
\hline 0 & 0 & 1 & 1 & 0 & 0 & 4 & 2 \\
\hline 5 & 3 & 7 & 2 & 0 & 7 & 3 & 8 \\
\hline 0 & 0 & 1 & 0 & 0 & 2 & 0 & 3 \\
\hline 0 & 0 & 0 & 0 & 0 & 2 & 0 & 4 \\
\hline 1 & 2 & 1 & 0 & 0 & 6 & 0 & 6 \\
\hline 2 & 0 & 0 & 0 & 0 & 0 & 0 & 4 \\
\hline
\end{tabular}


TABLE 2: (continued)

\begin{tabular}{|c|c|c|c|c|c|c|c|c|}
\hline C1QC & 2 & 0 & 1 & 1 & 0 & 1 & 2 & 3 \\
\hline C1RA & 0 & 0 & 0 & 0 & 0 & 3 & 0 & 4 \\
\hline CS1A & 1 & 0 & 1 & 1 & 0 & 4 & 0 & 4 \\
\hline CYC & 0 & 0 & 1 & 0 & 0 & 2 & 0 & 3 \\
\hline FBLN3 & 0 & 0 & 0 & 0 & 0 & 8 & 1 & 5 \\
\hline ECM1 & 1 & 0 & 1 & 0 & 0 & 6 & 1 & 5 \\
\hline SODE & 1 & 0 & 0 & 0 & 0 & 1 & 0 & 6 \\
\hline EZRI & 0 & 0 & 0 & 0 & 0 & 3 & 0 & 0 \\
\hline FRIH & 1 & 5 & 4 & 1 & 0 & 2 & 0 & 2 \\
\hline FIBG & 16 & 11 & 16 & 11 & 0 & 29 & 6 & 33 \\
\hline FINC & 30 & 18 & 30 & 8 & 0 & 54 & 0 & 75 \\
\hline GPX3 & 3 & 1 & 4 & 2 & 0 & 6 & 0 & 7 \\
\hline HA10 & 0 & 0 & 1 & 0 & 0 & 10 & 0 & 10 \\
\hline HSP7C & 0 & 0 & 0 & 0 & 0 & 0 & 0 & 3 \\
\hline $\mathrm{H} 2 \mathrm{~A} 1 \mathrm{~F}(+5)$ & 0 & 0 & 0 & 0 & 0 & 3 & 0 & 3 \\
\hline H2B1B (+7) & 0 & 0 & 0 & 0 & 0 & 2 & 0 & 4 \\
\hline $\mathrm{H} 4$ & 1 & 0 & 0 & 0 & 0 & 4 & 0 & 5 \\
\hline HABP2 & 0 & 0 & 0 & 0 & 0 & 3 & 0 & 2 \\
\hline HVM06 & 0 & 0 & 0 & 0 & 0 & 2 & 2 & 4 \\
\hline IGKC & 6 & 5 & 4 & 3 & 0 & 2 & 3 & 2 \\
\hline KV2A7 & 3 & 2 & 2 & 2 & 0 & 2 & 1 & 2 \\
\hline LAC2 $(+1)$ & 4 & 2 & 2 & 0 & 0 & 2 & 0 & 2 \\
\hline IGJ & 4 & 3 & 3 & 2 & 0 & 2 & 2 & 3 \\
\hline ALS & 5 & 6 & 5 & 3 & 0 & 6 & 5 & 6 \\
\hline ITIH2 & 5 & 0 & 3 & 2 & 0 & 20 & 11 & 22 \\
\hline ITIH3 & 0 & 0 & 2 & 0 & 0 & 8 & 0 & 18 \\
\hline K1C14 & 0 & 0 & 0 & 3 & 0 & 1 & 0 & 0 \\
\hline K1C16 & 0 & 0 & 0 & 3 & 0 & 0 & 0 & 0 \\
\hline K1C17 & 0 & 1 & 8 & 14 & 0 & 0 & 0 & 0 \\
\hline K1C42 & 2 & 1 & 2 & 5 & 0 & 0 & 0 & 0 \\
\hline
\end{tabular}


TABLE 2: (continued)

\begin{tabular}{|c|c|c|c|c|c|c|c|c|}
\hline K2C5 & 0 & 1 & 2 & 6 & 0 & 0 & 0 & 2 \\
\hline K2C73 & 3 & 2 & 2 & 3 & 0 & 0 & 0 & 4 \\
\hline TRFL & 0 & 0 & 4 & 6 & 0 & 4 & 23 & 21 \\
\hline MUP1 & 16 & 13 & 1 & 14 & 0 & 1 & 1 & 1 \\
\hline MASP1 & 0 & 0 & 1 & 0 & 0 & 3 & 0 & 2 \\
\hline PERM & 0 & 0 & 0 & 0 & 0 & 0 & 2 & 3 \\
\hline MYH1 (+1) & 0 & 3 & 0 & 0 & 0 & 0 & 0 & 0 \\
\hline PI16 & 3 & 0 & 0 & 0 & 0 & 1 & 1 & 1 \\
\hline PLMN & 15 & 6 & 16 & 4 & 0 & 28 & 7 & 42 \\
\hline S10A8 & 0 & 0 & 0 & 0 & 0 & 1 & 3 & 2 \\
\hline S10A9 & 0 & 0 & 2 & 4 & 0 & 3 & 9 & 11 \\
\hline THRB & 4 & 0 & 3 & 1 & 0 & 22 & 0 & 22 \\
\hline SAA1 & 0 & 0 & 0 & 0 & 0 & 0 & 5 & 9 \\
\hline SAA2 & 0 & 0 & 0 & 0 & 0 & 0 & 0 & 5 \\
\hline SAMP & 1 & 1 & 0 & 1 & 0 & 1 & 0 & 5 \\
\hline TFR1 & 0 & 0 & 0 & 0 & 0 & 3 & 0 & 2 \\
\hline TKT & 0 & 0 & 0 & 1 & 0 & 1 & 3 & 7 \\
\hline TPM1 & 0 & 0 & 0 & 0 & 0 & 1 & 0 & 5 \\
\hline TPM2 & 0 & 0 & 5 & 0 & 0 & 4 & 0 & 1 \\
\hline VTNC & 6 & 1 & 8 & 1 & 0 & 3 & 0 & 11 \\
\hline KAD1 & 0 & 0 & 0 & 0 & 1 & 3 & 0 & 1 \\
\hline ADIPO & 4 & 2 & 4 & 2 & 1 & 4 & 1 & 3 \\
\hline $\mathrm{ACE}$ & 3 & 1 & 2 & 0 & 1 & 2 & 1 & 2 \\
\hline APOA4 & 18 & 4 & 18 & 7 & 1 & 22 & 15 & 28 \\
\hline APOC1 & 0 & 0 & 2 & 0 & 1 & 4 & 3 & 3 \\
\hline CRP & 2 & 0 & 2 & 2 & 1 & 3 & 1 & 4 \\
\hline $\mathrm{CAH} 2$ & 6 & 4 & 4 & 2 & 1 & 5 & 3 & 3 \\
\hline CBPQ & 1 & 3 & 3 & 2 & 1 & 2 & 1 & 2 \\
\hline F13B & 0 & 0 & 1 & 0 & 1 & 11 & 8 & 10 \\
\hline C1QA & 1 & 0 & 1 & 0 & 1 & 0 & 3 & 2 \\
\hline
\end{tabular}


TABLE 2: (continued)

\begin{tabular}{|c|c|c|c|c|c|c|c|c|}
\hline $\mathrm{CO} 2$ & 0 & 0 & 0 & 0 & 1 & 4 & 1 & 3 \\
\hline FRIL1 & 0 & 8 & 1 & 0 & 1 & 3 & 0 & 1 \\
\hline FIBA & 28 & 17 & 21 & 17 & 1 & 44 & 7 & 54 \\
\hline GSTM1 & 0 & 0 & 0 & 0 & 1 & 3 & 0 & 0 \\
\hline G3P & 2 & 0 & 0 & 4 & 1 & 2 & 0 & 5 \\
\hline HGFA & 0 & 0 & 0 & 0 & 1 & 8 & 4 & 6 \\
\hline IGHA & 7 & 3 & 6 & 1 & 1 & 4 & 5 & 7 \\
\hline ITIH1 & 3 & 1 & 5 & 1 & 1 & 13 & 11 & 19 \\
\hline K2C6A & 1 & 4 & 7 & 14 & 1 & 1 & 0 & 0 \\
\hline LUM & 4 & 3 & 4 & 3 & 1 & 4 & 4 & 3 \\
\hline MUP20 & 5 & 5 & 5 & 3 & 1 & 3 & 2 & 2 \\
\hline MUP3 & 3 & 2 & 4 & 2 & 1 & 2 & 1 & 2 \\
\hline MDHC & 0 & 1 & 0 & 0 & 1 & 3 & 0 & 2 \\
\hline MBL1 & 1 & 1 & 2 & 0 & 1 & 3 & 4 & 5 \\
\hline MYH4 & 0 & 0 & 27 & 9 & 1 & 17 & 8 & 6 \\
\hline PHLD & 3 & 1 & 4 & 2 & 1 & 15 & 10 & 14 \\
\hline PGM1 & 0 & 5 & 1 & 0 & 1 & 3 & 2 & 0 \\
\hline PGAM2 & 0 & 0 & 1 & 0 & 1 & 3 & 1 & 0 \\
\hline AMBP & 4 & 2 & 2 & 2 & 1 & 6 & 3 & 7 \\
\hline SEPP1 & 1 & 1 & 1 & 0 & 1 & 3 & 4 & 5 \\
\hline SAA4 & 4 & 2 & 2 & 1 & 1 & 7 & 4 & 8 \\
\hline PON1 & 4 & 3 & 5 & 2 & 1 & 9 & 6 & 10 \\
\hline ENOA & 1 & 2 & 0 & 6 & 2 & 3 & 1 & 5 \\
\hline APOE & 8 & 7 & 13 & 8 & 2 & 21 & 8 & 18 \\
\hline СВРВ2 & 7 & 8 & 8 & 7 & 2 & 5 & 5 & 8 \\
\hline FIBB & 25 & 13 & 20 & 9 & 2 & 38 & 14 & 48 \\
\hline G6PI & 1 & 5 & 4 & 1 & 2 & 4 & 1 & 4 \\
\hline HEP2 & 0 & 0 & 0 & 0 & 2 & 2 & 5 & 2 \\
\hline MUP2 & 3 & 3 & 3 & 2 & 2 & 3 & 2 & 2 \\
\hline MYG & 0 & 1 & 0 & 0 & 2 & 6 & 0 & 0 \\
\hline
\end{tabular}


TABLE 2: (continued)

\begin{tabular}{|c|c|c|c|c|c|c|c|c|}
\hline ZPI & 1 & 2 & 3 & 1 & 2 & 5 & 4 & 10 \\
\hline TETN & 0 & 0 & 0 & 0 & 2 & 2 & 3 & 3 \\
\hline APOA2 & 6 & 4 & 5 & 6 & 3 & 6 & 6 & 6 \\
\hline ACTBL & 1 & 1 & 0 & 0 & 3 & 0 & 0 & 1 \\
\hline CELA1 & 1 & 1 & 1 & 1 & 3 & 4 & 2 & 1 \\
\hline FA12 & 2 & 1 & 6 & 0 & 3 & 14 & 10 & 14 \\
\hline CO8G & 2 & 1 & 3 & 1 & 3 & 6 & 5 & 6 \\
\hline CFAD & 8 & 7 & 7 & 7 & 3 & 6 & 6 & 7 \\
\hline GRN & 0 & 0 & 0 & 0 & 3 & 0 & 2 & 1 \\
\hline ITIH4 & 0 & 0 & 0 & 0 & 3 & 6 & 15 & 20 \\
\hline IL1AP & 2 & 0 & 3 & 0 & 3 & 6 & 4 & 6 \\
\hline LDHA & 2 & 4 & 4 & 1 & 3 & 10 & 3 & 5 \\
\hline TIMP1 & 0 & 0 & 0 & 0 & 3 & 0 & 2 & 1 \\
\hline PGRP2 & 0 & 0 & 0 & 0 & 3 & 2 & 4 & 4 \\
\hline PROP & 8 & 4 & 8 & 0 & 3 & 11 & 5 & 10 \\
\hline SPA3N & 2 & 0 & 2 & 1 & 3 & 7 & 12 & 27 \\
\hline TPIS & 2 & 8 & 5 & 2 & 3 & 8 & 2 & 5 \\
\hline CTRB1 & 0 & 0 & 0 & 0 & 4 & 0 & 0 & 0 \\
\hline $\mathrm{CBG}$ & 6 & 8 & 7 & 5 & 4 & 6 & 4 & 9 \\
\hline HBB 1 & 10 & 6 & 4 & 4 & 4 & 9 & 6 & 7 \\
\hline PRVA & 0 & 2 & 0 & 0 & 4 & 1 & 0 & 0 \\
\hline PPIA & 2 & 2 & 2 & 1 & 4 & 4 & 2 & 3 \\
\hline PGK1 & 0 & 8 & 1 & 1 & 4 & 5 & 3 & 1 \\
\hline KPYM & 3 & 12 & 13 & 3 & 4 & 11 & 8 & 13 \\
\hline SSLP1 & 0 & 0 & 0 & 0 & 4 & 2 & 5 & 2 \\
\hline TCO2 & 0 & 0 & 0 & 0 & 4 & 1 & 2 & 1 \\
\hline ZA2G & 1 & 0 & 1 & 0 & 4 & 8 & 10 & 10 \\
\hline ANGT & 4 & 4 & 5 & 3 & 5 & 8 & 9 & 7 \\
\hline CATB & 3 & 1 & 4 & 2 & 5 & 2 & 5 & 8 \\
\hline HPT & 11 & 8 & 11 & 9 & 5 & 8 & 9 & 19 \\
\hline
\end{tabular}


TABLE 2: (continued)

\begin{tabular}{|c|c|c|c|c|c|c|c|c|}
\hline LIFR & 11 & 3 & 10 & 2 & 5 & 11 & 11 & 8 \\
\hline LYZ2 & 0 & 0 & 0 & 0 & 5 & 1 & 4 & 3 \\
\hline RET4 & 0 & 0 & 1 & 0 & 5 & 7 & 8 & 7 \\
\hline SPA3M & 2 & 1 & 1 & 1 & 5 & 20 & 8 & 21 \\
\hline SPIKL & 0 & 0 & 0 & 0 & 5 & 2 & 4 & 1 \\
\hline ALBU & 8 & 8 & 27 & 11 & 5 & 30 & 22 & 24 \\
\hline A1AG1 & 15 & 13 & 12 & 11 & 6 & 13 & 10 & 14 \\
\hline CATL1 & 0 & 0 & 0 & 0 & 6 & 1 & 5 & 1 \\
\hline CO8A & 3 & 1 & 4 & 0 & 6 & 15 & 14 & 18 \\
\hline $\mathrm{CO} 8 \mathrm{~B}$ & 4 & 0 & 6 & 0 & 6 & 16 & 14 & 16 \\
\hline DNS2B & 0 & 0 & 0 & 0 & 6 & 0 & 2 & 0 \\
\hline HBA & 9 & 5 & 5 & 5 & 6 & 10 & 7 & 7 \\
\hline MA2B1 & 0 & 0 & 0 & 0 & 6 & 2 & 1 & 1 \\
\hline LIPP & 0 & 0 & 0 & 0 & 6 & 3 & 2 & 0 \\
\hline ENOB & 0 & 5 & 2 & 2 & 7 & 12 & 3 & 2 \\
\hline CEAMA & 0 & 0 & 0 & 0 & 7 & 1 & 4 & 2 \\
\hline $\mathrm{CO} 5$ & 7 & 1 & 17 & 3 & 7 & 24 & 22 & 29 \\
\hline $\mathrm{CO} 9$ & 2 & 0 & 1 & 0 & 7 & 12 & 10 & 15 \\
\hline IGHM & 29 & 27 & 25 & 18 & 7 & 24 & 20 & 24 \\
\hline ISK1 & 0 & 0 & 0 & 0 & 7 & 5 & 7 & 5 \\
\hline $\mathrm{A} 2 \mathrm{AP}$ & 2 & 0 & 2 & 0 & 8 & 11 & 10 & 13 \\
\hline $\mathrm{APOH}$ & 8 & 3 & 10 & 3 & 8 & 17 & 14 & 18 \\
\hline ALDOA & 5 & 17 & 20 & 7 & 8 & 23 & 15 & 14 \\
\hline MUP6 (+2) & 2 & 2 & 16 & 2 & 8 & 13 & 14 & 13 \\
\hline PEDF & 8 & 6 & 7 & 3 & 8 & 9 & 10 & 8 \\
\hline IC1 & 5 & 1 & 5 & 2 & 9 & 10 & 12 & 11 \\
\hline TTHY & 14 & 15 & 12 & 13 & 9 & 13 & 11 & 12 \\
\hline B2MG & 1 & 1 & 1 & 1 & 10 & 5 & 8 & 5 \\
\hline CYTC & 0 & 0 & 0 & 0 & 10 & 2 & 10 & 1 \\
\hline EGFR & 9 & 2 & 9 & 2 & 10 & 16 & 18 & 18 \\
\hline
\end{tabular}


TABLE 2: (continued)

\begin{tabular}{|c|c|c|c|c|c|c|c|c|}
\hline FETUB & 6 & 3 & 4 & 4 & 10 & 14 & 14 & 16 \\
\hline PATE4 & 0 & 0 & 0 & 0 & 10 & 6 & 10 & 6 \\
\hline A1AT1 & 4 & 2 & 10 & 2 & 11 & 17 & 4 & 17 \\
\hline CAH3 & 2 & 16 & 7 & 6 & 11 & 15 & 7 & 9 \\
\hline CEL2A & 0 & 0 & 0 & 0 & 11 & 3 & 5 & 3 \\
\hline CFAI & 9 & 2 & 7 & 4 & 11 & 23 & 22 & 23 \\
\hline KLKB1 & 4 & 0 & 8 & 1 & 11 & 25 & 20 & 24 \\
\hline SVS5 & 0 & 0 & 0 & 0 & 12 & 6 & 14 & 7 \\
\hline UROK & 0 & 0 & 0 & 0 & 12 & 1 & 7 & 0 \\
\hline HRG & 11 & 4 & 13 & 4 & 13 & 19 & 18 & 24 \\
\hline KNG1 & 13 & 7 & 14 & 8 & 13 & 20 & 17 & 21 \\
\hline SVS6 & 0 & 0 & 0 & 0 & 13 & 7 & 13 & 9 \\
\hline A1AT5 & 14 & 9 & 12 & 11 & 15 & 20 & 16 & 19 \\
\hline FETUA & 14 & 7 & 11 & 5 & 15 & 20 & 16 & 21 \\
\hline $\mathrm{CO} 4 \mathrm{~B}$ & 3 & 1 & 8 & 2 & 15 & 32 & 17 & 46 \\
\hline A1AT2 & 45 & 17 & 14 & 15 & 16 & 43 & 16 & 46 \\
\hline ICA & 17 & 7 & 13 & 5 & 16 & 31 & 27 & 33 \\
\hline AFAM & 22 & 23 & 23 & 15 & 18 & 29 & 21 & 27 \\
\hline ANT3 & 18 & 14 & 16 & 15 & 18 & 28 & 24 & 30 \\
\hline KCRM & 0 & 28 & 2 & 9 & 18 & 23 & 4 & 9 \\
\hline SVS4 & 0 & 0 & 0 & 0 & 18 & 12 & 18 & 13 \\
\hline GDN & 0 & 0 & 0 & 0 & 19 & 0 & 14 & 0 \\
\hline SPA3K & 15 & 16 & 14 & 16 & 20 & 9 & 22 & 9 \\
\hline APOA1 & 37 & 26 & 30 & 27 & 21 & 39 & 33 & 44 \\
\hline AMYP & 6 & 9 & 7 & 2 & 21 & 23 & 16 & 14 \\
\hline CERU & 62 & 32 & 53 & 45 & 25 & 61 & 46 & 63 \\
\hline CFAB & 26 & 13 & 24 & 13 & 25 & 45 & 34 & 47 \\
\hline CFAH & 36 & 19 & 37 & 17 & 26 & 59 & 46 & 63 \\
\hline GELS & 13 & 6 & 12 & 7 & 26 & 36 & 33 & 34 \\
\hline VTDB & 38 & 25 & 30 & 26 & 28 & 48 & 39 & 55 \\
\hline
\end{tabular}


TABLE 2: (continued)

\begin{tabular}{|c|c|c|c|c|c|c|c|c|}
\hline EST1C & 32 & 29 & 31 & 27 & 29 & 34 & 31 & 34 \\
\hline HEMO & 40 & 33 & 38 & 27 & 32 & 39 & 39 & 40 \\
\hline MUG1 & 56 & 37 & 50 & 36 & 35 & 87 & 71 & 77 \\
\hline QSOX1 & 0 & 0 & 0 & 0 & 35 & 12 & 27 & 10 \\
\hline A1AT4 & 17 & 39 & 37 & 38 & 39 & 17 & 41 & 18 \\
\hline PZP & 0 & 0 & 0 & 0 & 52 & 110 & 102 & 106 \\
\hline $\mathrm{CO} 3$ & 79 & 52 & 91 & 64 & 66 & 107 & 98 & 135 \\
\hline TRFE & 117 & 105 & 91 & 105 & 91 & 117 & 102 & 110 \\
\hline
\end{tabular}

TABLE 3: Peritoneal ascites were analyzed by proteomics

\begin{tabular}{lcccccccc} 
& \multicolumn{4}{c}{ Sham } & \multicolumn{4}{c}{ Plasma (2) } \\
Accession Num- & $\mathbf{0}$ & $\mathbf{0 . 5}$ & $\mathbf{4}$ & $\mathbf{2 4}$ & $\mathbf{0}$ & $\mathbf{0 . 5}$ & $\mathbf{4}$ & $\mathbf{2 4}$ \\
ber & $\mathbf{h r}$ & $\mathbf{h r}$ & $\mathbf{h r}$ & $\mathbf{h r}$ & $\mathbf{h r}$ & $\mathbf{h r}$ & $\mathbf{h r}$ & $\mathbf{h r}$ \\
6PGD & 0 & 0 & 0 & 0 & 0 & 0 & 0 & 8 \\
ACTS (+3) & 0 & 0 & 2 & 1 & 0 & 0 & 3 & 2 \\
A1AT3 & 7 & 6 & 0 & 6 & 0 & 0 & 9 & 0 \\
A2M & 102 & 77 & 92 & 77 & 0 & 0 & 0 & 0 \\
ANXA5 & 0 & 2 & 0 & 1 & 0 & 3 & 1 & 2 \\
APOC1 & 0 & 0 & 2 & 0 & 0 & 0 & 3 & 2 \\
\hline ENOB & 0 & 5 & 2 & 2 & 0 & 5 & 4 & 12 \\
CRP & 2 & 0 & 2 & 2 & 0 & 0 & 2 & 4 \\
\hline CAMP & 0 & 0 & 0 & 0 & 0 & 0 & 1 & 3 \\
CHIL3 & 0 & 0 & 1 & 1 & 0 & 0 & 2 & 13 \\
FA10 & 0 & 0 & 1 & 0 & 0 & 1 & 0 & 5 \\
F13A & 0 & 0 & 0 & 0 & 0 & 1 & 1 & 7 \\
CO3A1 & 2 & 0 & 0 & 0 & 0 & 0 & 0 & 3 \\
C1RA & 0 & 0 & 0 & 0 & 0 & 1 & 1 & 4 \\
\hline SODE & 1 & 0 & 0 & 0 & 0 & 1 & 0 & 3 \\
\hline FBLN1 & 0 & 0 & 0 & 0 & 0 & 0 & 1 & 5 \\
\hline HBB1 & 10 & 6 & 4 & 4 & 0 & 5 & 5 & 5 \\
LAC2 (+1) & 4 & 2 & 0 & 0 & 0 & 2 & 0 & 1 \\
\hline PLAK & 0 & 0 & 0 & 2 & 0 & 2 & 0 & 6 \\
\hline TRFL & 0 & 0 & 4 & 6 & 0 & 0 & 12 & 35 \\
\hline
\end{tabular}


TABLE 3: (continued)

\begin{tabular}{lcccccccc} 
LKHA4 & 0 & 0 & 0 & 0 & 0 & 0 & 0 & 5 \\
MASP1 & 0 & 0 & 1 & 0 & 0 & 1 & 1 & 4 \\
MBL2 & 0 & 0 & 0 & 0 & 0 & 2 & 1 & 3 \\
PERM & 0 & 0 & 0 & 0 & 0 & 0 & 0 & 4 \\
MYG & 0 & 1 & 0 & 0 & 0 & 7 & 3 & 5 \\
MYL1 & 0 & 0 & 0 & 0 & 0 & 0 & 0 & 4 \\
MYH1 & 0 & 3 & 0 & 0 & 0 & 0 & 4 & 0 \\
MYH4 $(+1)$ & 0 & 0 & 27 & 9 & 0 & 0 & 49 & 28 \\
PGRP2 & 0 & 0 & 0 & 0 & 0 & 1 & 1 & 3 \\
NGP & 0 & 0 & 0 & 0 & 0 & 0 & 0 & 6 \\
NUCB1 & 0 & 0 & 1 & 0 & 0 & 1 & 0 & 4 \\
PRVA & 0 & 2 & 0 & 0 & 0 & 2 & 2 & 6 \\
GP1BA & 0 & 0 & 0 & 0 & 0 & 1 & 1 & 4 \\
S10A8 & 0 & 0 & 0 & 0 & 0 & 0 & 4 & 3 \\
S10A9 & 0 & 0 & 2 & 4 & 0 & 0 & 11 & 7 \\
RNAS4 & 0 & 0 & 0 & 0 & 0 & 0 & 0 & 3 \\
SAA1 & 0 & 0 & 0 & 0 & 0 & 0 & 0 & 6 \\
TETN & 0 & 0 & 0 & 0 & 0 & 1 & 1 & 3 \\
TALDO & 0 & 0 & 0 & 0 & 0 & 0 & 1 & 5 \\
TPM1 & 0 & 0 & 0 & 0 & 0 & 3 & 12 & 3 \\
TPM2 & 0 & 0 & 5 & 0 & 0 & 1 & 4 & 6 \\
ENOA & 1 & 2 & 0 & 6 & 1 & 0 & 0 & 3 \\
\hline ANXA2 & 0 & 0 & 0 & 0 & 1 & 0 & 0 & 4 \\
\hline APOB & 0 & 0 & 8 & 0 & 1 & 14 & 7 & 45 \\
\hline B2MG & 1 & 1 & 1 & 1 & 1 & 4 & 2 & 2 \\
CALM & 0 & 0 & 0 & 0 & 1 & 1 & 1 & 3 \\
CHLE & 0 & 0 & 0 & 0 & 1 & 3 & 1 & 5 \\
F13B & 0 & 0 & 1 & 0 & 1 & 4 & 3 & 9 \\
COF1 & 0 & 0 & 0 & 0 & 1 & 0 & 0 & 3 \\
C1QA & 1 & 0 & 1 & 0 & 1 & 1 & 1 & 3 \\
C1QB & 1 & 1 & 1 & 0 & 1 & 1 & 0 & 3 \\
C1QC & 2 & 0 & 1 & 1 & 1 & 1 & 1 & 3 \\
ECM1 & 1 & 0 & 1 & 0 & 1 & 3 & 5 & 11 \\
GSHR & 0 & 0 & 0 & 0 & 1 & 1 & 0 & 6 \\
HA10 & 0 & 1 & 0 & 1 & 2 & 1 & 7 \\
HGFA & & & & & & & & 5 \\
& 0 & 0 & 0 & 1 & 2 & 2 & 5 \\
\hline
\end{tabular}


TABLE 3: (continued)

\begin{tabular}{lcccccccc} 
KV2A7 & 3 & 2 & 2 & 2 & 1 & 2 & 2 & 1 \\
LAC1 & 1 & 1 & 1 & 0 & 1 & 1 & 1 & 3 \\
IBP4 & 0 & 0 & 0 & 0 & 1 & 1 & 0 & 3 \\
K1C17 & 0 & 1 & 8 & 14 & 1 & 0 & 0 & 0 \\
K1C42 & 2 & 1 & 2 & 5 & 1 & 2 & 0 & 2 \\
MUP1 & 16 & 13 & 1 & 14 & 1 & 1 & 1 & 0 \\
\hline MBL1 & 1 & 1 & 2 & 0 & 1 & 3 & 4 & 5 \\
LCAT & 0 & 0 & 2 & 0 & 1 & 1 & 1 & 5 \\
PSA6 & 1 & 1 & 1 & 0 & 1 & 1 & 1 & 3 \\
SAMP & 1 & 1 & 0 & 1 & 1 & 1 & 2 & 5 \\
SPRL1 & 0 & 0 & 0 & 0 & 1 & 3 & 3 & 4 \\
QSOX1 & 0 & 0 & 0 & 0 & 1 & 1 & 2 & 7 \\
THBG & 0 & 0 & 0 & 0 & 1 & 0 & 1 & 3 \\
1433Z & 0 & 0 & 0 & 0 & 2 & 2 & 1 & 6 \\
SAHH & 0 & 1 & 0 & 0 & 2 & 3 & 2 & 1 \\
ACE & 3 & 1 & 2 & 0 & 2 & 4 & 4 & 4 \\
ANXA3 & 0 & 0 & 0 & 0 & 2 & 3 & 0 & 0 \\
APOC3 & 2 & 2 & 2 & 2 & 2 & 3 & 3 & 3 \\
BTD & 1 & 1 & 1 & 1 & 2 & 2 & 2 & 6 \\
C4BPA & 2 & 0 & 1 & 1 & 2 & 6 & 5 & 10 \\
CO1A1 & 1 & 2 & 1 & 0 & 2 & 3 & 3 & 5 \\
FCN1 & 0 & 0 & 1 & 0 & 2 & 3 & 3 & 5 \\
G6PI & 1 & 5 & 4 & 1 & 2 & 4 & 5 & 9 \\
\hline GSTM1 (+2) & 0 & 0 & 0 & 0 & 2 & 4 & 3 & 3 \\
HBA & 9 & 5 & 5 & 5 & 2 & 4 & 4 & 4 \\
IGJ & 4 & 3 & 3 & 2 & 2 & 2 & 3 & 2 \\
ITIH3 & 0 & 0 & 2 & 0 & 2 & 6 & 3 & 11 \\
K1C16 & 0 & 0 & 0 & 3 & 2 & 1 & 0 & 1 \\
K2C1 & 1 & 1 & 2 & 2 & 2 & 2 & 1 & 3 \\
K2C73 & 3 & 2 & 2 & 3 & 2 & 2 & 2 & 1 \\
LDHA & 2 & 4 & 4 & 1 & 2 & 3 & 5 & 6 \\
MDHC & 0 & 1 & 0 & 0 & 2 & 2 & 1 & 3 \\
PI16 & 3 & 0 & 0 & 0 & 2 & 2 & 1 & 2 \\
PGM1 & 5 & 1 & 0 & 2 & 6 & 3 & 5 \\
REG3B & 0 & 0 & 0 & 2 & 3 & 2 & 2 \\
SAA4 & & & & & & & & 8
\end{tabular}


TABLE 3: (continued)

\begin{tabular}{lcccccccc} 
TFR1 & 0 & 0 & 0 & 0 & 2 & 4 & 1 & 5 \\
AMY1 & 2 & 2 & 2 & 1 & 3 & 5 & 6 & 5 \\
APOD & 1 & 0 & 1 & 0 & 3 & 2 & 3 & 4 \\
CO8G & 2 & 1 & 3 & 1 & 3 & 4 & 5 & 7 \\
CO9 & 2 & 0 & 1 & 0 & 3 & 4 & 4 & 7 \\
KCRM & 0 & 28 & 2 & 9 & 3 & 25 & 20 & 15 \\
G3P & 2 & 0 & 0 & 4 & 3 & 0 & 1 & 4 \\
\hline H2B1A (+11) & 0 & 0 & 0 & 0 & 3 & 0 & 0 & 1 \\
H4 & 1 & 0 & 0 & 0 & 3 & 0 & 0 & 4 \\
KV3A1 (+1) & 0 & 0 & 0 & 0 & 3 & 2 & 3 & 3 \\
IBP6 & 1 & 0 & 0 & 0 & 3 & 3 & 3 & 0 \\
K1C10 & 0 & 0 & 0 & 0 & 3 & 4 & 2 & 6 \\
MUP2 & 3 & 3 & 3 & 2 & 3 & 3 & 3 & 2 \\
RET4 & 0 & 0 & 1 & 0 & 3 & 2 & 3 & 5 \\
SEPP1 & 1 & 1 & 1 & 0 & 3 & 1 & 2 & 6 \\
SH3L3 & 1 & 1 & 0 & 1 & 3 & 1 & 1 & 2 \\
ADIPO & 4 & 2 & 4 & 2 & 4 & 5 & 4 & 5 \\
A2AP & 2 & 0 & 2 & 0 & 4 & 5 & 5 & 6 \\
CAH2 & 6 & 4 & 4 & 2 & 4 & 5 & 4 & 4 \\
CYC & 0 & 0 & 1 & 0 & 4 & 3 & 3 & 2 \\
FRIH & 1 & 5 & 4 & 1 & 4 & 3 & 3 & 3 \\
IL1AP & 2 & 0 & 3 & 0 & 4 & 4 & 3 & 8 \\
K22E & 1 & 2 & 1 & 2 & 4 & 4 & 0 & 6 \\
LUM & 4 & 3 & 4 & 3 & 4 & 4 & 6 & 7 \\
MUP3 & 3 & 2 & 4 & 2 & 4 & 8 & 5 & 7 \\
PGK1 & 0 & 8 & 1 & 1 & 4 & 7 & 4 & 9 \\
AMBP & 4 & 2 & 2 & 2 & 4 & 4 & 5 & 7 \\
WDR1 & 0 & 0 & 0 & 0 & 4 & 1 & 0 & 3 \\
CLUS & 5 & 3 & 7 & 2 & 5 & 6 & 5 & 14 \\
CO8A & 3 & 1 & 4 & 0 & 5 & 8 & 8 & 16 \\
CO8B & 4 & 0 & 6 & 0 & 5 & 6 & 10 & 14 \\
GPX3 & 3 & 1 & 4 & 2 & 5 & 5 & 6 & 4 \\
IGHA & 7 & 3 & 6 & 1 & 5 & 6 & 6 & 4 \\
IGKC & 5 & 4 & 3 & 5 & 3 & 2 & 5 \\
K2C6A & & & 2 & 0 & 5 & 3 & 3 & 6 \\
\hline CSF1R & & & & & & 3 \\
\hline
\end{tabular}


TABLE 3: (continued)

\begin{tabular}{|c|c|c|c|c|c|c|c|c|}
\hline PPIA & 2 & 2 & 2 & 1 & 5 & 6 & 4 & 5 \\
\hline $\mathrm{IC} 1$ & 5 & 1 & 5 & 2 & 5 & 6 & 5 & 8 \\
\hline ZPI & 1 & 2 & 3 & 1 & 5 & 7 & 7 & 8 \\
\hline SPA3M & 2 & 1 & 1 & 1 & 5 & 3 & 5 & 16 \\
\hline ANGT & 4 & 4 & 5 & 3 & 6 & 7 & 7 & 8 \\
\hline CBPN & 4 & 2 & 5 & 3 & 6 & 7 & 7 & 9 \\
\hline CPN2 & 2 & 3 & 6 & 3 & 6 & 10 & 7 & 11 \\
\hline CBPQ & 1 & 3 & 3 & 2 & 6 & 7 & 4 & 5 \\
\hline FRIL1 (+1) & 0 & 8 & 1 & 0 & 6 & 4 & 2 & 1 \\
\hline ITIH1 & 3 & 1 & 5 & 1 & 6 & 7 & 9 & 21 \\
\hline LIFR & 11 & 3 & 10 & 2 & 6 & 10 & 9 & 17 \\
\hline LYZ2 & 0 & 0 & 0 & 0 & 6 & 1 & 3 & 2 \\
\hline THRB & 4 & 0 & 3 & 1 & 6 & 10 & 9 & 15 \\
\hline KPYM & 3 & 12 & 13 & 3 & 6 & 16 & 15 & 18 \\
\hline SPA3N & 2 & 0 & 2 & 1 & 6 & 4 & 4 & 6 \\
\hline SODC & 1 & 2 & 1 & 1 & 6 & 4 & 1 & 3 \\
\hline ZA2G & 1 & 0 & 1 & 0 & 6 & 6 & 6 & 6 \\
\hline APOA2 & 6 & 4 & 5 & 6 & 7 & 7 & 7 & 4 \\
\hline CAH3 & 2 & 16 & 7 & 6 & 7 & 13 & 13 & 15 \\
\hline СATB & 3 & 1 & 4 & 2 & 7 & 5 & 5 & 8 \\
\hline GUAD & 2 & 2 & 0 & 0 & 7 & 0 & 3 & 8 \\
\hline ITIH4 & 0 & 0 & 0 & 0 & 7 & 0 & 1 & 12 \\
\hline VTNC & 6 & 1 & 8 & 1 & 7 & 10 & 8 & 10 \\
\hline CD5L & 4 & 3 & 5 & 2 & 8 & 7 & 5 & 8 \\
\hline FETUB & 6 & 3 & 4 & 4 & 8 & 12 & 11 & 13 \\
\hline ALDOA & 5 & 17 & 20 & 7 & 8 & 18 & 17 & 18 \\
\hline ALS & 5 & 6 & 5 & 3 & 8 & 9 & 8 & 10 \\
\hline MUP20 & 5 & 5 & 5 & 3 & 8 & 4 & 3 & 4 \\
\hline PON1 & 4 & 3 & 5 & 2 & 8 & 7 & 8 & 11 \\
\hline TPIS & 2 & 8 & 5 & 2 & 8 & 11 & 9 & 10 \\
\hline CBPB2 & 7 & 8 & 8 & 7 & 9 & 9 & 9 & 9 \\
\hline FA12 & 2 & 1 & 6 & 0 & 9 & 10 & 9 & 11 \\
\hline $\mathrm{CO} 5$ & 7 & 1 & 17 & 3 & 9 & 17 & 18 & 40 \\
\hline CFAD & 8 & 7 & 7 & 7 & 9 & 7 & 7 & 6 \\
\hline ITIH2 & 5 & 0 & 3 & 2 & 9 & 18 & 18 & 16 \\
\hline $\mathrm{K} 1 \mathrm{C} 14$ & 0 & 0 & 0 & 3 & 9 & 7 & 2 & 11 \\
\hline
\end{tabular}


TABLE 3: (continued)

\begin{tabular}{|c|c|c|c|c|c|c|c|}
\hline $\mathrm{K} 2 \mathrm{C} 5$ & 0 & 1 & 2 & 6 & 9 & 12 & 0 \\
\hline PROP & 8 & 4 & 8 & 0 & 9 & 11 & 12 \\
\hline PEDF & 8 & 6 & 7 & 3 & 10 & 10 & 8 \\
\hline KLKB1 & 4 & 0 & 8 & 1 & 10 & 13 & 17 \\
\hline CFAI & 9 & 2 & 7 & 4 & 11 & 13 & 15 \\
\hline HPT & 11 & 8 & 11 & 9 & 11 & 11 & 9 \\
\hline $\operatorname{ACTB}(+1)$ & 5 & 4 & 6 & 6 & 12 & 10 & 11 \\
\hline A1AT1 & 4 & 2 & 10 & 2 & 12 & 13 & 5 \\
\hline APOE & 8 & 7 & 13 & 8 & 12 & 19 & 17 \\
\hline $\mathrm{APOH}$ & 8 & 3 & 10 & 3 & 12 & 15 & 13 \\
\hline EGFR & 9 & 2 & 9 & 2 & 12 & 14 & 13 \\
\hline PHLD & 3 & 1 & 4 & 2 & 12 & 12 & 15 \\
\hline TTHY & 14 & 15 & 12 & 13 & 12 & 11 & 11 \\
\hline FETUA & 14 & 7 & 11 & 5 & 13 & 16 & 16 \\
\hline HRG & 11 & 4 & 13 & 4 & 14 & 23 & 15 \\
\hline CBG & 6 & 8 & 7 & 5 & 15 & 18 & 11 \\
\hline SPA3K & 15 & 16 & 14 & 16 & 15 & 16 & 15 \\
\hline A1AG1 & 15 & 13 & 12 & 11 & 16 & 16 & 17 \\
\hline A1AT5 & 14 & 9 & 12 & 11 & 16 & 18 & 15 \\
\hline APOA4 & 18 & 4 & 18 & 7 & 16 & 18 & 18 \\
\hline KNG1 & 13 & 7 & 14 & 8 & 16 & 18 & 23 \\
\hline MUP6 (+2) & 2 & 2 & 16 & 2 & 16 & 16 & 15 \\
\hline FIBG & 16 & 11 & 16 & 11 & 17 & 28 & 22 \\
\hline ICA & 17 & 7 & 13 & 5 & 17 & 26 & 25 \\
\hline A1AT4 & 17 & 39 & 37 & 38 & 19 & 19 & 17 \\
\hline $\mathrm{CO} 4 \mathrm{~B}$ & 3 & 1 & 8 & 2 & 19 & 24 & 18 \\
\hline GELS & 13 & 6 & 12 & 7 & 21 & 24 & 26 \\
\hline FIBA & 28 & 17 & 21 & 17 & 22 & 32 & 30 \\
\hline IGHM & 29 & 27 & 25 & 18 & 22 & 23 & 18 \\
\hline AMYP & 6 & 9 & 7 & 2 & 22 & 14 & 14 \\
\hline PLMN & 15 & 6 & 16 & 4 & 22 & 27 & 36 \\
\hline FIBB & 25 & 13 & 20 & 9 & 26 & 30 & 32 \\
\hline ANT3 & 18 & 14 & 16 & 15 & 27 & 33 & 26 \\
\hline AFAM & 22 & 23 & 23 & 15 & 30 & 30 & 28 \\
\hline APOA1 & 37 & 26 & 30 & 27 & 32 & 36 & 33 \\
\hline HEMO & 40 & 33 & 38 & 27 & 32 & 35 & 37 \\
\hline
\end{tabular}


TABLE 3: (continued)

\begin{tabular}{|c|c|c|c|c|c|c|c|c|}
\hline EST1C & 32 & 29 & 31 & 27 & 34 & 35 & 35 & 25 \\
\hline CFAB & 26 & 13 & 24 & 13 & 35 & 30 & 33 & 32 \\
\hline CFAH & 36 & 19 & 37 & 17 & 36 & 39 & 47 & 49 \\
\hline ALBU & 8 & 8 & 27 & 11 & 38 & 36 & 37 & 31 \\
\hline A1AT2 & 45 & 17 & 14 & 15 & 43 & 45 & 44 & 10 \\
\hline FINC & 30 & 18 & 30 & 8 & 48 & 51 & 43 & 74 \\
\hline VTDB & 38 & 25 & 30 & 26 & 48 & 55 & 53 & 31 \\
\hline CERU & 62 & 32 & 53 & 45 & 57 & 57 & 63 & 52 \\
\hline MUG1 & 56 & 37 & 50 & 36 & 63 & 70 & 73 & 65 \\
\hline PZP & 0 & 0 & 0 & 0 & 81 & 106 & 106 & 74 \\
\hline $\mathrm{CO} 3$ & 79 & 52 & 91 & 64 & 110 & 114 & 108 & 97 \\
\hline TRFE & 117 & 105 & 91 & 105 & 113 & 116 & 114 & 72 \\
\hline
\end{tabular}
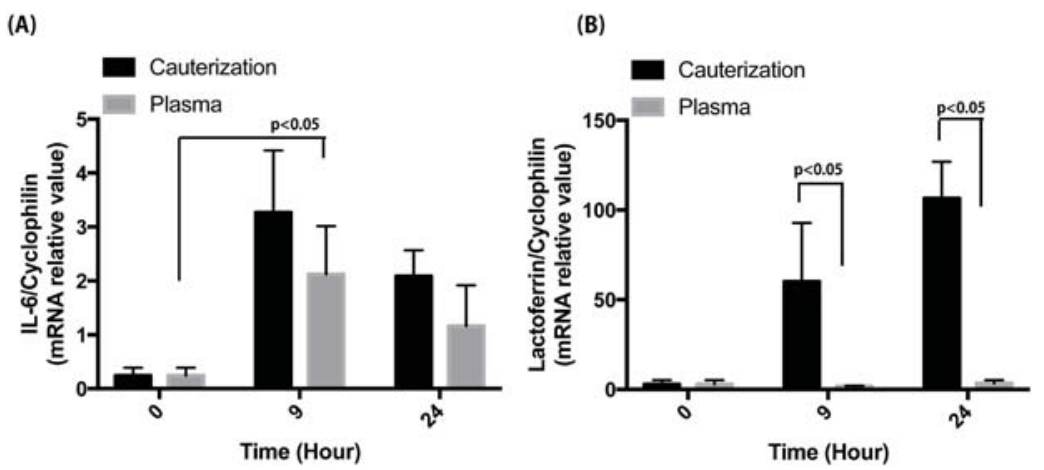

FIG. 7: Inflammatory effects due to plasma irradiation were very weak compared to cauterization. (a) IL-6 mRNA level in cecum was quantified by real-time PCR. IL-6 was induced at $9 \mathrm{hr}$ after plasma irradiation. IL-6 level after plasma irradiation was not significantly different from the level induced by cauterization, but the trend of IL- 6 in plasma irradiation appeared smaller than IL-6 in cauterization. (b) Lactoferrin (LTF) mRNA was strongly induced when cauterizing the cecum. Plasma irradiation showed little induction of LTF. ( $N=6$ in each experiment).

The results of inflammatory proteins identified by proteomics in ascites, such as lactoferrin, serum amyloid protein, inter- $\alpha$-trypsin inhibitor, serine protease inhibitor, and apolipoprotein B released in peritoneal cavity, raised the possibility that are candidates associated with adhesion formation. ${ }^{45}$ Quantitative mass spectroscopy ${ }^{46}$ may help to reveal the mechanism underlying adhesion formation in the abdominal cavity.

Collectively, plasma irradiation affects less inflammatory response to abdominal tissues (Fig. 8).

Volume 7, Issue 4, 2017 


\section{Adhesion mouse model}

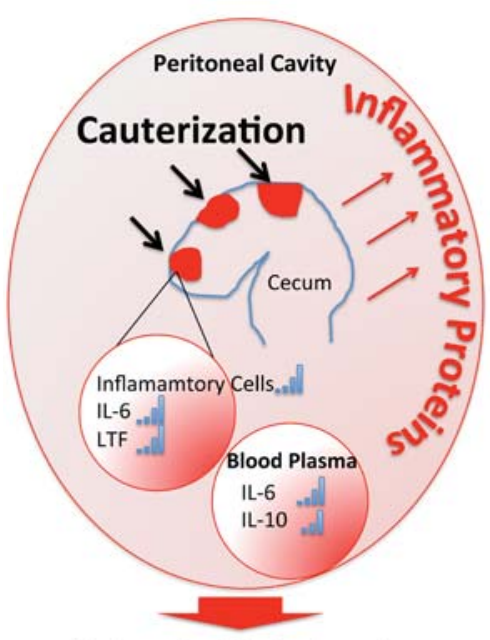

Postoperative Adhesion Formation

\section{Plasma irradiation}

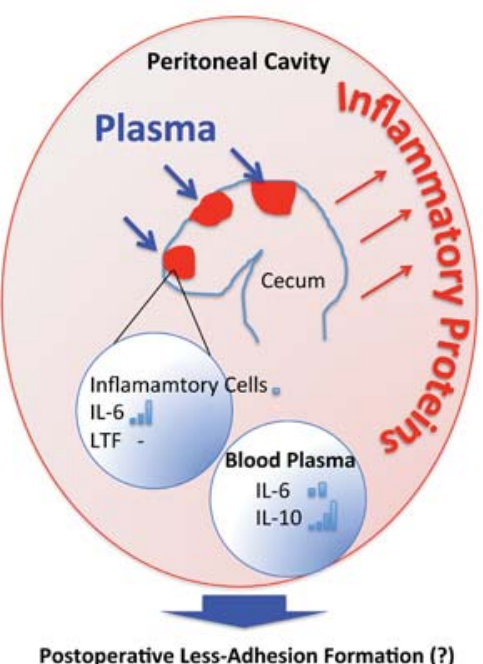

Strong induction

=1대

Weak induction

FIG. 8: Schematic diagram of our results summarize plasma irradiation effects. Even though plasma irradiation of the tissue caused inflammation in the peritoneal cavity, few inflammatory cells were infiltrated in local tissue by plasma irradiation, which might be associated with whether adhesion occurs.

\section{ACKNOWHAGMENT}

The Contribution of the Grant-in-Aid for Scientific Research on Innovative Areas "Plasma Medical Science Innovation (24108007)" from the Ministry of Education, Culture, Sports, Science and Technology of Japan is gratefully acknowledged.

\section{REFERENCES}

1. National Institutes of Health. Abdominal adhesions. [serial on Internet] 2013 Sept. Available from: https://www.niddk.nih.gov/health-information/digestive-diseases/abdominal-adhesions.

2. Wiseman DM. Disorders of adhesions or adhesion-related disorder: monolithic entities or part of something bigger-CAPPS? Semin Reprod Med. 2008;26(4):356-68.

3. Arung W, Meurisse M, Detry O. Pathophysiology and prevention of postoperative peritoneal adhesions. World J Gastroenterol. 2011;17(41):4545-53. 
4. Erkek AB, Remzi FH, Hammel JP, Akyuz M, Fazio VW. Effect of small bowel obstruction on functional outcome and quality of life in patients with ileal pouch-anal anastomosis: 10-year follow-up study. J Gastroenterol Hepatol. 2008;23(1):119-25.

5. Duron JJ, Silva NJ, du Montcel ST, Berger A, Muscari F, Hennet H, Veyrieres M, Hay JM. Adhesive postoperative small bowel obstruction: incidence and risk factors of recurrence after surgical treatment: a multicenter prospective study. Ann Surg. 2006;244(5):750-7.

6. Bruggmann D, Tchartchian G, Wallwiener M, Munstedt K, Tinneberg HR, Hackethal A. Intra-abdominal adhesions: definition, origin, significance in surgical practice, and treatment options. Dtsch Arztebl Int. 2010;107(44):769-75.

7. Awonuga AO, Fletcher NM, Saed GM, Diamond MP. Postoperative adhesion development following cesarean and open intra-abdominal gynecological operations: a review. Reprod Sci. 2011;18(12):116685 .

8. Hindocha A, Beere L, Dias S, Watson A, Ahmad G. Adhesion prevention agents for gynaecological surgery: an overview of Cochrane reviews. Cochrane Database Syst Rev. 2015;1:CD011254.

9. Attard JA, MacLean AR. Adhesive small bowel obstruction: epidemiology, biology and prevention. Can J Surg. 2007;50(4):291-300.

10. Bhatta N, Isaacson K, Flotte T, Schiff I, Anderson RR. Injury and adhesion formation following ovarian wedge resection with different thermal surgical modalities. Lasers Surg Med. 1993;13(3):344-52.

11. Rodrigo R. Prevention of postoperative atrial fibrillation: novel and safe strategy based on the modulation of the antioxidant system. Front Physiol. 2012;3:93.

12. Vetere PF, Lazarou G, Mondesir C, Wei K, Khullar P, Ogden L. Strategies to minimize adhesion formation after surgery. JSLS. 2011;15(3):350-4.

13. Javaherzadeh M, Shekarchizadeh A, Kafaei M, Mirafshrieh A, Mosaffa N, Sabet B. Effects of intraperitoneal administration of simvastatin in prevention of postoperative intra-abdominal adhesion formation in animal model of rat. Bull Emerg Trauma. 2016;4(3):156-60.

14. Yeo Y, Kohane DS. Polymers in the prevention of peritoneal adhesions. Eur J Pharm Biopharm. 2008;68(1):57-66.

15. Matoba M, Hashimoto A, Tanzawa A, Orikasa T, Ikeda J, Iwame Y, Ozamoto Y, Abe R, Miyamoto H, Yoshida C, Hashimoto T, Torii H, Takamori H, Morita S, Tsujimoto H, Hagiwara A. Prevention of polyglycolic acid-induced peritoneal adhesions using alginate in a rat model. Biomed Res Int. 2015;2015:403413.

16. Haensig M, Mohr FW, Rastan AJ. Bioresorbable adhesion barrier for reducing the severity of postoperative cardiac adhesions: focus on REPEL-CV ${ }^{\circledR}$. Med Devices (Auckl). 2011;4:17-25.

17. Stommel MW, Strik C, ten Broek RP, van Goor H. Efficacy and safety of the C-Qur Film Adhesion Barrier for the prevention of surgical adhesions (CLIPEUS Trial): study protocol for a randomized controlled trial. Trials. 2014;15:378.

18. Coelho Junior ER, Costa LO, Alencar AV, Barbosa AP, Pinto FC, Aguiar JL. Prevention of peritoneal adhesion using a bacterial cellulose hydrogel, in experimental study. Acta Cir Bras. 2015;30(3):194-8.

19. Lang R, Baumann P, Jauch KW, Schmoor C, Weis C, Odermatt E, Knaebel HP. A prospective, randomised, controlled, double-blind phase I-II clinical trial on the safety of A-Part Gel as adhesion prophylaxis after major abdominal surgery versus non-treated group. BMC Surg. 2010;10:20.

20. Ahmad G, O'Flynn H, Hindocha A, Watson A. Barrier agents for adhesion prevention after gynaecological surgery. Cochrane Database Syst Rev. 2015(4):CD000475.

21. Olmarker K. Reduction of adhesion formation and promotion of wound healing after laminectomy by pharmacological inhibition of pro-inflammatory cytokines: an experimental study in the rat. Eur Spine J. 2010;19(12):2117-21.

22. Maciver AH, McCall M, James Shapiro AM. Intra-abdominal adhesions: cellular mechanisms and strategies for prevention. Int J Surg. 2011;9(8):589-94.

23. Cheong YC, Laird SM, Li TC, Shelton JB, Ledger WL, Cooke ID. Peritoneal healing and adhesion formation/reformation. Hum Reprod Update. 2001;7(6):556-66.

Volume 7, Issue 4, 2017 
24. Tanaka H, Nakamura K, Mizuno M, Ishikawa K, Takeda K, Kajiyama H, Utsumi F, Kikkawa F, Hori M. Non-thermal atmospheric pressure plasma activates lactate in Ringer's solution for anti-tumor effects. Sci Rep. 2016;6:36282.

25. Yamada H, Kato S, Kim J, Kiyama S, Fujiwara M, Itagaki H, Okazaki T, Ikehara S, Nakanishi H, Shimizu N, Ikehara Y. Spectroscopy of reactive species produced by low-energy atmospheric-pressure plasma on conductive target material surface. J Phys D: Appl Phys. 2016;49(39):1-11.

26. Ikehara S, Ishikawa K, Akimoto Y, Yamaguchi T, Yamagishi M, Kim J, Ueda M, Ikeda JI, Nakanishi H, Shimizu N, Hori M, Ikehara Y. Plasma blood coagulation without involving the activation of platelets and coagulation factors. Plasma Process Polym. 2015;12(12):1348-53.

27. Daroz LR, Lopes JB, Dallan LA, Campana-Filho SP, Moreira LF, Stolf NA. Prevention of postoperative pericardial adhesions using thermal sterile carboxymethyl chitosan. Rev Bras Cir Cardiovasc. 2008;23(4):480-7.

28. Kosaka H, Yoshimoto T, Yoshimoto T, Fujimoto J, Nakanishi K. Interferon- $\gamma$ is a therapeutic target molecule for prevention of postoperative adhesion formation. Nat Med. 2008;14(4):437-41.

29. Sakakita H, Ikehara Y. Irradiation experiments on a mouse using a mild-plasma generator for medical applications. Plasma Fusion Res. 2010;5:S2117.

30. Aarons CB, Cohen PA, Gower A, Reed KL, Leeman SE, Stucchi AF, Becker JM. Statins (HMG-CoA reductase inhibitors) decrease postoperative adhesions by increasing peritoneal fibrinolytic activity. Ann Surg. 2007;245(2):176-84.

31. Oncel M, Remzi FH, Connor J, Fazio VW. Comparison of cecal abrasion and multiple-abrasion models in generating intra-abdominal adhesions for animal studies. Tech Coloproctol. 2005;9(1):29-33.

32. Zhang W, Bi B, Oldroyd RG, Lachmann PJ. Neutrophil lactoferrin release induced by IgA immune complexes differed from that induced by cross-linking of Fca receptors (FcaR) with a monoclonal antibody, MIP8a. Clin Exp Immunol. 2000;121(1):106-11.

33. Akimoto Y, Ikehara S, Yamaguchi T, Kim J, Kawakami H, Shimizu N, Hori M, Sakakita H, Ikehara Y. Galectin expression in healing wounded skin treated with low-temperature plasma: comparison with treatment by electronical coagulation. Arch Biochem Biophys. 2016;605:86-94.

34. Hung YW, Lee LT, Peng YC, Chang CT, Wong YK, Tung KC. Effect of a nonthermal-atmospheric pressure plasma jet on wound healing: an animal study. J Chin Med Assoc. 2016;79(6):320-8.

35. Haertel B, von Woedtke T, Weltmann KD, Lindequist U. Non-thermal atmospheric-pressure plasma possible application in wound healing. Biomol Ther (Seoul). 2014;22(6):477-90.

36. Brun P, Pathak S, Castagliuolo I, Palu G, Brun P, Zuin M, Cavazzana R, Martines E. Helium generated cold plasma finely regulates activation of human fibroblast-like primary cells. PLoS One. 2014;9(8):e104397.

37. Morfill GE, Shimizu T, Steffes B, Schmidt H-U. Nosocomial infections-a new approach towards preventive medicine using plasmas. New J Phys. 2009;11:1-10.

38. Chi L, Li Y, Stehno-Bittel L, Gao J, Morrison DC, Stechschulte DJ, Dileepan KN. Interleukin-6 production by endothelial cells via stimulation of protease-activated receptors is amplified by endotoxin and tumor necrosis factor- $\alpha$. J Interferon Cytokine Res. 2001;21(4):231-40.

39. Moreno A, Villar ML, Camara C, Luque R, Cespon C, Gonzalez-Porque P, Roy G, Lopez-Jimenez J, Bootello A, Santiago ER. Interleukin-6 dimers produced by endothelial cells inhibit apoptosis of Bchronic lymphocytic leukemia cells. Blood. 2001;97(1):242-9.

40. Sundararaj KP, Samuvel DJ, Li Y, Sanders JJ, Lopes-Virella MF, Huang Y. Interleukin-6 released from fibroblasts is essential for up-regulation of matrix metalloproteinase-1 expression by U937 macrophages in coculture: cross-talking between fibroblasts and U937 macrophages exposed to high glucose. J Biol Chem. 2009;284(20):13714-24.

41. Moodley YP, Misso NL, Scaffidi AK, Fogel-Petrovic M, McAnulty RJ, Laurent GJ, Thompson PJ, Knight DA. Inverse effects of interleukin-6 on apoptosis of fibroblasts from pulmonary fibrosis and normal lungs. Am J Respir Cell Mol Biol. 2003;29(4):490-8.

42. Verbeek W, Lekstrom-Himes J, Park DJ, Dang PM, Vuong PT, Kawano S, Babior BM, Xanthopoulos 
K, Koeffler HP. Myeloid transcription factor C/EBPE is involved in the positive regulation of lactoferrin gene expression in neutrophils. Blood. 1999;94(9):3141-50.

43. Peranteau WH, Zhang L, Muvarak N, Badillo AT, Radu A, Zoltick PW, Liechty KW. IL-10 overexpression decreases inflammatory mediators and promotes regenerative healing in an adult model of scar formation. J Invest Dermatol. 2008;128(7):1852-60.

44. Hunter CA, Jones SA. IL-6 as a keystone cytokine in health and disease. Nat Immunol. 2015;16(5):44857.

45. Catena F, Di Saverio S, Coccolini F, Ansaloni L, De Simone B, Sartelli M, Van Goor H. Adhesive small bowel adhesions obstruction: evolutions in diagnosis, management and prevention. World J Gastrointest Surg. 2016;8(3):222-31.

46. Bery A, Leung F, Smith CR, Diamandis EP, Kulasingam V. Deciphering the ovarian cancer ascites fluid peptidome. Clin Proteomics. 2014;11(1):13.

Volume 7, Issue 4, 2017 
